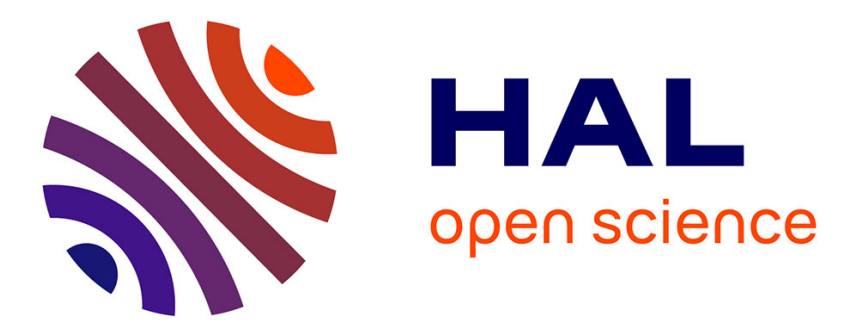

\title{
Hybrid polarizable simulations of a conventional hydrophobic polyelectrolyte. Toward a theoretical tool for green science innovation
}

Michel Masella, Alina Crudu, Fabien Léonforté

\section{- To cite this version:}

Michel Masella, Alina Crudu, Fabien Léonforté. Hybrid polarizable simulations of a conventional hydrophobic polyelectrolyte. Toward a theoretical tool for green science innovation. Journal of Chemical Physics, 2021, 155 (11), pp.114903. 10.1063/5.0056508 . hal-03366342

\section{HAL Id: hal-03366342 \\ https://hal.science/hal-03366342}

Submitted on 5 Oct 2021

HAL is a multi-disciplinary open access archive for the deposit and dissemination of scientific research documents, whether they are published or not. The documents may come from teaching and research institutions in France or abroad, or from public or private research centers.
L'archive ouverte pluridisciplinaire HAL, est destinée au dépôt et à la diffusion de documents scientifiques de niveau recherche, publiés ou non, émanant des établissements d'enseignement et de recherche français ou étrangers, des laboratoires publics ou privés. 


\title{
Hybrid polarizable simulations of a
}

\section{conventional Hydrophobic PolyElectrolyte.}

\section{Towards a theoretical tool for Green Science}

\section{innovation.}

\author{
Michel Masella, ${ }^{* \dagger}$ Alina Crudu, ${ }^{\ddagger}$ and Fabien Léonforté*, \\ $†$ †aboratoire de Biologie Structurale et Radiobiologie, Service de Bioénergétique, Biologie \\ Structurale et Mécanismes, Institut Joliot, CEA Saclay, F-91191 Gif sur Yvette Cedex, \\ France \\ $\ddagger$ L’Oréal Research $\mathscr{G}$ Innovation, France
}

E-mail: michel.masella@cea.fr; fabien.leonforte@rd.loreal.com 


\begin{abstract}
Hybrid modeling approaches based on all-atom force fields to handle a solute and coarse grained models to account for the solvent are promising numerical tools that can be used to understand the properties of large and multi components solutions and thus to speed up the development of new industrial products that obey the standard of green and sustainable chemistry. Here we discuss the ability of a full polarizable hybrid approach coupled to a standard Molecular Dynamics scheme to model the behavior in aqueous phase and at infinite dilution conditions of a standard hydrophobic polyelectrolyte polymer whose charge is neutralized by explicit counter ions. Beyond the standard picture of a polyelectrolyte behavior governed by an interplay between opposite intra polyelectrolyte and inter polyelectrolyte/counter ion Coulombic effects, our simulations show the key role played by both intra solute polarization effects and long range solute/solvent electrostatics to stabilize compact globular conformations of that polyelectrolyte. Our full polarizable hybrid modeling approach is thus a new theoretical tool well suited to be used in digital strategies for accelerating innovation for Green Sciences, for instance.
\end{abstract}




\section{${ }_{18} 1$ Introduction}

Developing new products that obey the standards of green and sustainable chemistry with eco-sustainable ingredients is presently a major goal of many industries ranging from chemistry, oil manufacturers, personal care or cosmetics. However, because of the complexity of most of the solutions/emulsions that these industries use, the development of new, safe and biodegradable products is a particularly challenging task. For instance, standard oil-in-water emulsions, for cosmetic purpose for instance, comprise several components like neutral/charged flexible macromolecules, anionic/zwitterionic surfactants as well as salts and alcohols, and lots of efforts focus today in making such formula more and more natural by substituting historical compounds by naturally-derived molecules, e.g. polysaccharides ${ }^{1}$. However these kinds of emulsions correspond to complex multi-phasic systems whose properties are highly sensitive to the substitution/addition of new compounds and most of the inherent mechanisms that are involved in systems under such conditions are still poorly understood.

To substitute a component in a solution, a strategy consists in understanding how it interacts with its partners and to select a substitute able to play a similar role. This can be a priori achieved from experimental data, in particular microscopic structural ones or from physico-chemically equivalent parameters matching. However investigating experimentally the microscopic structures of complex molecular assemblies in solution is still a challenging task in modern chemistry and materials science $^{2,3}$. Regarding macromolecular polymeric systems for instance, Atomic Force Microscope, AFM, imaging technique is able to provide mesoscopic pictures of them in various environments at best at the $10 \mathrm{~nm}$ scale ${ }^{4-7}$. Small Angle Neutron and X-ray Scattering, SANS/SAXS, techniques are also commonly used to investigate polymer-based solutions ${ }^{8-11}$ even if the interpretation of their data may be controversial. ${ }^{12}$ To tackle out the lack of experimental data, molecular simulations are a 
valuable alternative. Since the nineties, computer Monte-Carlo, MC, and Molecular Dynamics, MD, simulations started to play an important role to understand the properties of polymer-based solutions, see Refs ${ }^{13,14}$ for instance. These simulations are performed using different theoretical approaches, from pure all-atom schemes for which all the system components (solute, solvent and counter ions) are modeled at the same level of accuracy to hybrid approaches for which the solvent can be accounted for implicitly. For instance in 2009 Yethiraj and co-workers reported simulations performed using pure all-atom and hybrid approaches of simple Hydrophobic PolyElectrolyte, HPE, systems comprising at most 384 beads ${ }^{15,16}$. Today with the on-going increase of the available computational resources, more realistic all-atom simulations are commonly performed to investigate the properties of polyelectrolytes in aqueous phase. Besides simulations based on standard Lennard-Jones, LJ, potentials (see the recent studies Refs. ${ }^{17-20}$ ), MD simulations at the 100 ns scale of large hydrated polyelectrolyte systems (comprising up to 250k atoms) performed using standard pairwise force fields (and lattice-based Ewald summation techniques to account for long range electrostatics) are now regularly reported, see Refs. ${ }^{21-23}$ for instance.

Despite the impressive progress of molecular modeling techniques, simulations of complex multi-components solutions/emulsions are still not routinely performed. That arises from the atomic size of realistic emulsions that are at the very least one to two order of magnitude larger than the systems simulated in the above studies. Moreover most of the simulations performed today rely on standard additive force fields that are acknowledged for their inability in accurately modeling charged systems in a polar medium like water, from single ion ${ }^{24-27}$ to ionic liquids ${ }^{28,29}$ and all hydrated polyelectrolyte systems ${ }^{30}$. That results from the inability of additive force fields to model the inherent non-pairwise character of microscopic polarization, a pivotal force to understand the interaction involving charged species, see Fig. 1.

Here we present a simulation work based on a new polarizable hybrid molecu- 
lar simulation approach a priori well suited for accelerating innovation with digital strategies for Green Sciences. That approach relies on modeling interactions among non-solvent atoms using the all atoms polarizable force field TCPEp, which is based on a polarizable induced dipole moment approach $^{31}$, like the one of Fig. 1. However polarizable force fields are computationally demanding, not only because of the polarization computational cost (in particular as using Drude oscillators ${ }^{32}$ ) but also because usually such force fields also consider more sophisticated interaction potentials to model electrostatic effects (like the force field AMOEBA that accounts for atomic static dipoles and quadrupoles ${ }^{33}$ ) or short range electronic density reorganization phenomena, like TCPEp ${ }^{31}$.

To perform efficiently MD simulations in aqueous phase by means of polarizable force fields, one of us proposed to handle water using the multi-level Polarizable Pseudo Particle, $\mathbf{P P P}^{l}$, coarse-grained, CG, approach ${ }^{34-36}$. $\mathbf{P P P}^{l}$ belongs to the macroscopic Density Functional Theory field. However as it retains the notion of particles, it also allows for an explicit solvent representation that is recommended for a realistic modeling of polyelectrolytes ${ }^{18}$. Moreover as $\mathbf{P P P}^{l}$ relies on a polarizable induced dipole moment approach, it can be readily coupled to all-atom induced dipole moment-based polarizable force fields like AMOEBA and TCPEp ${ }^{37}$. Two important features of the $\mathbf{P P P}^{l}$ approach are (1) to readily allow simulations in infinite dilution conditions and (2) to permit the modulation of the spatial range of the solvent electrostatic effects on a solute. That second feature allows the modulation the apparent solvent dielectric constant by taking constant the intensity of local interactions between the solvent particles and the solute ${ }^{36}$. As compared to standard all-atom simulations based on periodic condition schemes, the $\mathbf{P P P}^{l}$ approach thus allows one to investigate the properties of a HPE in water-like solutions that are fully disentangled from solute concentration effects, as well as to discuss the role of solvent macroscopic properties (in particular the magnitude of its dielectric constant). 
The current work aims at showing the ability of the hybrid $\mathbf{P P P}^{l} /$ TCPEp approach to model the complex components of industrial solutions and thus at paving the way in developing new digital workflows allowing to speed up the development of Natural products based on the knowledge of conventional ones. To this aim, we first focus on investigating the behavior of a generic mid-size HPE copolymer that is commonly used in conventional chemical industry for water/wastewater treatments, personal care as well as for clean and renewable energy production ${ }^{38-42}$.

The HPE we consider, that is schematically depicted in Fig. 2, possesses a fraction $f_{c}$ of charged groups per copolymer unit equals to 0.7 , and independent from usual $\mathrm{pH}$ conditions. We denote that Polyelectrolyte as P70 (because of its total charge : $+70 e)$ and we simulate it in two water-like solutions at infinite dilution conditions by means of MD simulations and of our hybrid approach : $\mathbf{P 7 0}$ and its $\mathrm{Cl}^{-}$counter ions are modeled by the all-atom polarizable TCPEp force field whereas the water-like environments are handled by the $\mathbf{P P P}^{l} \mathrm{CG}$ approach. In particular we simulated the P70 collapse from a linear conformation as dissolved in pure (i.e. salt free) $\mathbf{P P P}^{l}$ box that comprised about $0.9 \mathrm{M}$ solvent particles. The dielectric constant of that environment is one order of magnitude smaller than for liquid water. Then we investigated the effect of the solvent dielectric constant on the stability of the P70 collapsed structures by simulating them embedded in a eight times more extended $\mathbf{P P P}^{l}$ medium whose dielectric constant is close to the liquid water one.

Less attention has been paid to investigate the role of polarization on HPEs than on bio-molecular polyelectrolytes like DNA/RNA ${ }^{43-45}$ and charged polypeptides ${ }^{46}$. HPE properties in aqueous phase are usually inferred to arise from a interplay of microscopic interactions, namely intra HPE Coulombic repulsion and effective attraction resulting from hydrophobic effects ${ }^{47,48}$. We thus simulated a non-polarizable $\mathbf{P} 70$ in our two $\mathbf{P P P}^{l}$ media in order to further discuss the role of polarization on hydrated HPEs beyond the standard Coulombic picture ${ }^{49-53}$.

As far as we know no experimental data regarding the conformational behav- 
The all-atom polarizable force field TCPEp ${ }^{31}$ is based on a total potential energy $U$ of a molecular system decomposed into a sum of six contributions

$$
U=U^{r e p}+U^{q q^{\prime}}+U^{p o l}+U^{l h}+U^{d i s p}+U^{i n t r a} .
$$

The last energy term is the sum of the standard stretching, bending, improper torsional and dihedral torsional energy terms modeling the interactions among covalently bonded atoms. The first two terms correspond to the inter atomic short range repulsion and to Coulombic interactions

$$
\begin{gathered}
U^{r e p}=\sum_{i=1}^{N} \sum_{j>i}^{N^{*}} A_{i j} \exp \left(-B_{i j} r_{i j}\right), \\
U^{q q^{\prime}}=\sum_{i=1}^{N} \sum_{j>i}^{N^{*}} \frac{q_{i} q_{j}}{4 \pi \varepsilon_{0} r_{i j}} .
\end{gathered}
$$

$r_{i j}$ is the distance between atoms $i$ and $j$, the $q_{i}$ s are the static charges located on atomic centers, and $A_{i j}$ and $B_{i j}$ are adjustable parameters. $N$ is the total number of atoms within the molecular system and the superscript ${ }^{*}$ indicates that the corresponding sum includes only pairs of atoms separated by more than two chemical 
bonds. The repulsive term is truncated for distances $r_{i j}$ greater than $5 \AA$.

The polarization energy term $U^{p o l}$ is based on an induced dipole moment approach. Induced dipole moments $\left\{\mathbf{p}_{i}\right\}_{1 \leq i \leq N_{\mu}}$ are introduced, one per polarizable non-hydrogen atom. They obey

$$
\mathbf{p}_{i}=\alpha_{i} \cdot\left(\mathbf{E}_{i}^{q}+\sum_{j=1}^{N_{\mu}^{*}} \mathbf{T}_{i j} \cdot \mathbf{p}_{j}\right)
$$

The static charge electric field $\mathbf{E}_{i}^{q}$ acting on a polarizable atom $i$ is generated only by the charges of the $N_{\mathbf{E}}$ surrounding atoms belonging to charged or dipolar chemical moieties. The $N_{\mu}$ and $N_{\mathbf{E}}$ sets do not necessarily match to each other. Let us consider the molecule $\mathrm{HO}-\mathrm{CH}_{2}-\mathrm{CH}_{2}-\mathrm{CH}_{2} \mathrm{CH}_{2}-\mathrm{NH}_{3}{ }^{+}$: only its first three atoms $\mathrm{HO}-\mathrm{C}$ (alcoholic moiety) and its last four ones $\mathrm{C}-\mathrm{NH}_{3}{ }^{+}$(cationic head) belong to the $N_{\mathbf{E}}$ set, and only its carbon and oxygen atoms are polarizable centers. $\alpha_{i}$ is the isotropic polarizability of the polarizable atom $i$ and $\mathbf{T}_{i j}$ is the dipolar interaction tensor. The static electric fields and dipolar tensors include short range Thole-like damping functions ${ }^{55}$ that vanish for interatomic distances greater than $5 \AA$. The set of Eqs. 2 is iteratively solved and the resulting polarization energy term $U^{\text {pol }}$ is

$$
U^{p o l}=\frac{1}{2} \sum_{i=1}^{N_{\mu}} \frac{\mathbf{p}_{i}^{2}}{\alpha_{i}}-\sum_{i=1}^{N_{\mu}} \mathbf{p}_{i} \cdot \mathbf{E}_{i}^{q}-\frac{1}{2} \sum_{i=1}^{N_{\mu}} \sum_{j=1}^{N_{\mu}^{*}} \mathbf{p}_{i} \mathbf{T}_{i j} \mathbf{p}_{j} .
$$

The term $U^{\text {disp }}$ models microscopic dispersion effects that are pivotal to describe hydrophobic alkyl chains. It is a sum of basic $C_{i j} / r_{i j}^{6}$ terms where the $C_{i j}(<0)$ are ajustable parameters. For our purpose only non-hydrogen atoms are considered as dispersion centers. To model particular electronic density reorganization effects (as those occurring within acrylamide hydrogen bonds), TCPEp also includes a set of short-range cooperative energy terms denoted $U^{l h}$ that are very close to the terms we introduce to model accurately water hydrogen bond networks ${ }^{56}$. For the present study such energy terms are taken into account to model the interactions among 
the P70 acrylamide moieties. However they play a negligible role in our simulations and they will not be further discussed.

\section{$2.2 \quad$ Force field parameters}

TCPEp parameters are adjusted to reproduce high-level ab-initio quantum data regarding a training set of small molecular systems (like the atomic charges that are assigned to reproduce the dipole moment of neutral molecules and the charges of charged molecules to meet those computed from the quantum Natural Population Analysis scheme ${ }^{57}$ ). P70 can be decomposed in a set of chemical groups for which we have already assigned accurate parameters ${ }^{35,36,58-60}$, at the exception of a reduced set of parameters regarding the torsional degrees of freedom of the dimethyldiallyl ammonium five membered ring that were specifically assigned for the present study according to the protocol detailed in Ref. ${ }^{59}$.

Regarding alkyl groups, their parameters (mainly repulsion and dispersion) were assigned to reproduce the quantum binding energy $\left(-0.5 \mathrm{kcal} \mathrm{mol}^{-1}\right)$ and geometry (a carbon/carbon equilibrium distance of $3.5 \AA$ ) of the methane dimer in gas phase as predicted by quantum $\operatorname{CCSD}(\mathrm{T})$ computations at the Complete Basis Set limit ${ }^{61}$. Because of the very weak permanent dipole in alkanes, we assume in the present study that only short range repulsion and dispersion govern the interactions involving alkyl groups in absence of external electric fields. We thus systematically neglect Coulombic interactions and static charge electric fields arising from alkyl moieties. However we consider the alkyl carbons as polarizable centers (their isotropic polarizability is set to $2.1 \AA^{3}$ ) and they undergo the static charge and/or induced dipole electric fields generated by all the chemical moieties surrounding them.

Lastly, we use no long range truncation scheme as computing Coulombic interactions and static charge electric fields regarding $\mathbf{P 7 0}$ and its counter ion cloud, whereas we truncate all their induced dipole/induced dipole interactions corresponding to distances larger than $12 \AA$. 
191

\subsection{The multi-level coarse-grained water model $\mathrm{PPP}^{l}$}

The main feature of the water coarse-grained approach $\mathbf{P} \mathbf{P} \mathbf{P}^{l 35-37}$ is to model a single three atomic water molecule or a set of water molecules by a single polarizable pseudo-particule (denoted as PPP) whose polarizability $\alpha_{s}$ obeys a Clausius-Mosotti relation:

$$
\alpha_{s}=\frac{\epsilon_{s}-1}{4 \pi \rho_{s} \epsilon_{s}}
$$

here, $\epsilon_{s}$ and $\rho_{s}$ are the dielectric constant and the particle density of liquid water (a quantity that is proportional to the inverse of the water molecular volume). $\alpha_{s}$ is thus a linear function of the PPP volume. Another important assumption is the use of the local approximation : the intensity of the induced dipole moment $\mathbf{p}_{j}^{s}$ corresponding to PPP $j$ is modulated only by the solute electric field $\mathbf{E}_{\text {solute }}^{j}$ acting on $j$, i.e. the PPPs don't polarize each other. However that can yield to largely overestimate solute/PPP polarization effects up to unphysical values in particular situations. To prevent such artefacts, $\mathbf{P P P} \mathbf{P}^{l}$ allows the $\mathbf{p}_{j}^{s}$ s to saturate according to $^{34}$

$$
\mathbf{p}_{j}^{s}=\mu_{s} \mathcal{L}\left(\frac{3 \alpha_{s} \mathbf{E}_{\text {solute }}^{j}}{\mu_{s}}\right) \frac{\mathbf{E}_{\text {solute }}^{j}}{\left|\mathbf{E}_{\text {solute }}^{j}\right|},
$$
cor

here $\mathcal{L}$ is the Langevin function and $\mu_{s}$ is the PPP saturation dipole value. The corresponding $\mathrm{PPP} /$ solute polarization energy is then

$$
U_{p s}^{p o l}=-\frac{\mu_{s}^{2}}{3 \alpha_{s}} \sum_{j=1}^{N_{s}} \ln \left[\frac{\sinh \left(3 \alpha_{s}\left|\mathbf{E}_{\text {solute }}^{j}\right| / \mu_{s}\right)}{3 \alpha_{s}\left|\mathbf{E}_{\text {solute }}^{j}\right| \mu_{s}}\right] .
$$

Here $N_{s}$ is the number of PPPs. Note that for both the above equations and weak solute electric fields, the linear regime corresponding to Eqs. 2-3 is recovered.

The polarizability $\alpha_{s}$ of the PPPs accounts not only for the solvent electronic polarization induced by the solute but also for the solvent orientational polarization ${ }^{34}$. 
As such $\alpha_{s}$ does not correspond to a standard atomic or a molecular polarizability (which models only the perturbation of an electronic cloud by an external electric field). Moreover the $\alpha_{s}$ allows one to consider PPPs of different sizes. As modeling a HPE by means of an all-atom force field, the natural choice is to set the size of all the PPPs to that of a single water molecule (that yields a PPP polarizability of 2.35 $\left.\AA^{3}\right)$. Moreover, within the $\mathbf{P P P}^{l}$ framework, we assume solute/solvent long range electrostatic contributions arising from neutral solute groups to be negligible. We thus systematically truncate both the induced dipole and static charge components of the corresponding electric fields $\mathbf{E}_{\text {solute }}^{j}$ for solute atom/PPP distances greater than the cut off distance $R_{\mathrm{cut}}^{\mathrm{pol}, 1}$.

However the above spherical truncation scheme not only yields large underestimation of ion hydration energies but also large artefacts in modeling ion association (see Ref. ${ }^{36}$ and references cited therein). To remediate both these drawbacks we proposed the multi-level scheme $\mathbf{P P P}^{l}$ to model efficiently the long-range electrostatic interactions between a charged solute and farther (and farther) solvent domains ${ }^{36}$. Within a shell lying at the vicinity of the charged solute (that shell extends to no more than $R_{\mathrm{cut}}^{\mathrm{pol}, 1}$ from any non hydrogen solute atom) that multi-level approach considers PPPs whose size matches that of a water molecule. These first solvent level PPPs $(l=1)$ undergo the static charge electric field generated by all the solute charged groups (and the truncated static and dipole electric field components arising from neutral groups). To model solute/solvent interactions at longer distances than $R_{\mathrm{cut}}^{\mathrm{pol}, 1}$, larger (and larger) PPPs are introduced according to the scheme shown in Fig. 3. These larger PPPs define higher level $(l>1)$ solvent shells. They undergo the static charge electric field generated by all the solute charged groups if they lie within a shell surrounding the solute and extending from $R_{\mathrm{cut}}^{\mathrm{pol}, l-1}$ to $R_{\mathrm{cut}}^{\mathrm{pol}, l}$ from it. The solute/solvent polarization term $U_{p s, l>1}^{\mathrm{pol}}$ corresponding to each $l>1$ PPP level is taken under the linear form 


$$
U_{p s, l>1}^{\mathrm{pol}}=\frac{1}{2} \sum_{i=1}^{N_{\mu}^{l>1}} \frac{\mathbf{p}_{i}^{2}}{\alpha_{s}^{l>1}}-\sum_{i=1}^{N_{\mu}^{l>1}} \mathbf{p}_{i} \cdot \mathbf{E}_{i}^{q} .
$$

Here, $N_{\mu}^{l>1}$ are the number of $l>1$ PPPs, $\alpha_{s}^{l>1}$ is their polarizability, according to Eq. 4 , and $\mathbf{E}_{i}^{q}$ is the static charge electric field generated by the solute charged groups on these PPPs.

Besides largely improving the modeling of ion hydration and ion association, we also showed that multi-level approach to allow an efficient modeling of particularly large and extended solvent domains at the $\mu \mathrm{m}$ scale and above ${ }^{36}$. For the present study, we consider two $\mathbf{P P P}^{l}$ solvent approaches. The first, denoted Short Range PPP, SRP, models the solvent by means of only first level PPPs and by setting the shell-based cut off distance $R_{\text {cut }}^{\mathrm{pol}, 1}$ to $12 \AA$. The second approach, denoted Long Range PPP, LRP, considers also a second level of PPPs made of particles that are 8 times larger than the first level ones. Their polarizability is thus $\alpha_{s, 2}=18.8 \AA^{3}$. The second level PPPs undergo the static charge electric field generated the P70 charged groups if these PPPs are located within a shell encompassing the HPE from $R_{\mathrm{cut}}^{\mathrm{pol}, 1}=12$ to $R_{\mathrm{cut}}^{\mathrm{pol}, 2}=143 \AA$. In an earlier study ${ }^{36}$ we showed the $\mathbf{S R P}$ approach to reinforce the association of oppositely charged ions and the repulsion of charge-like ions as compared to liquid water (the apparent dielectric constant of a first level PPP solution is one order of magnitude weaker than that of liquid water), whereas the LRP approach already provides a description of ion pairing agreeing with that expected in liquid water. The dielectric constant of the LRP medium is thus close to the liquid water one.

Within the multi level $\mathbf{P P P}^{l}$ approach, only electrostatic solute/l $>1$ PPPs interactions are taken into account. Regarding first level PPPs, their non-electrostatic interactions with solute atoms are modeled by means of a Lennard-Jones-like potential corresponding to a stronger repulsion ${ }^{37}$ to which is added a specific short range many-body term to prevent over populated first hydration shell at the vicinity of 
charged species ${ }^{36}$. The corresponding force field parameters for first level PPPs are assigned to reproduce both the first hydration shell structure and the hydration Gibbs energy of a training set of small solutes and ions ${ }^{36}$. For the present purpose we used an updated parameters that will be discussed elsewhere.

Interactions among first level PPPs are modeled using a standard additive LennardJones, LJ, energy term and a many-body term that is a function of the solvent local density at the vicinity of a $\mathrm{PPP}^{35}$. Both these terms are truncated for inter particle distances greater than $R_{\text {cut }}^{\mathrm{pp}}=7 \AA$ and their parameters were assigned to reproduce the liquid water density $\left(0.0331\right.$ molecule per $\left.\AA^{3}\right)$ and the two regimes of the energy corresponding to the creation of an empty cavity in water at ambient conditions according to the Lum-Chandler-Weeks theory of hydrophobicity ${ }^{62}$.

Interactions among second order PPPs are modeled using only a LJ term that is truncated for distances greater than $10 \AA$. The corresponding LJ radius is twice as large as for first order PPPs and the LJ intensity (denoted $\epsilon_{2}$ ) is one order of magnitude weaker than for the first level PPPs as in our original study ${ }^{36}$. In that original study we showed second level PPPs to be slightly over concentrated at their lower cut off distance as interacting with a single monovalent ion like $\mathrm{Na}^{+}$and $\mathrm{Cl}^{-}$. Because of the large total charge of the $\mathbf{P 7 0}(+70 e)$ and because of the overall large number of counter ions $\mathrm{Cl}^{-}$, our choice regarding the LJ parameter $\epsilon_{2}$ can lead to drawbacks like over organized P70/counter ions/second order PPPs structures. We will discuss that particular issue in the forthcoming Section 4.

Contrary to the recent approach proposed by Chremos and Douglas ${ }^{17,20,63}$, the use of only first level PPPs or in conjunction with second level ones allows the modulation of the apparent dielectric constant of the solvent without altering the short range solute/solvent interactions. Moreover our shell-based cutoff scheme also allows the modeling of solvation at infinite dilution conditions. In our $\mathbf{P P P}^{l}$ approach, a solute is embedded in a cubic box fulfilled with first level PPPs and that system is then embedded in a larger box made of second level PPPs and so on ${ }^{36}$. To maintain 
the PPP density within each box, the PPPs are allowed to interact with their own periodic images. As the long range interactions among PPPs are truncated, only the first 26 periodic box images are usually needed for that task. Regarding the solute, to model its solvation at infinite dilution conditions, one just needs to neglect the interactions between the solute and its own periodic images. That choice is made for both the solvent approaches SRP and LRP. However note that solute atoms can interact with PPP images if they lie close to the box boundaries.

Regarding SRP, ignoring the interactions between the solute and its own periodic images corresponds technically to model it as infinitively diluted. However we may also interpret that solute as embedded in a confined water cavity delimited by the shell of first order PPPs. That interpretation is supported by the modeling of the short range solute/PPP interactions using the same potential energy terms in both the SRP and LRP approaches.

We assume here first level PPPs (those who interact at short range with the solute) to be symmetric. Other choices are possible as considering a triatomic molecule holding a permanent dipole like the isolated water molecule. However we may note that the isotropic polarizability of Equation 4 allows to account for both the solvent orientational and electronic polarization induced by a solute ${ }^{34}$. Moreover modeling the solvent using triatomic molecules will lead the approach to be less computationally efficient, at least by factor 3 .

\subsection{Molecular Dynamics details}

MD simulations are performed at ambient conditions using the code POLARIS(MD) ${ }^{64}$. The Newtonian equations of motion are solved using a multiple-time-steps algorithm with two time steps: $0.25 \mathrm{fs}$ for intra-solute stretching, bending and improper torsional energy terms and $2 f s$ for all the other interactions. All the covalent $\mathrm{X}-\mathrm{H}$ bonds and $\mathrm{H}-\mathrm{X}-\mathrm{H}$ angles are constrained to their force field reference values by means of the iterative RATTLE procedure (the convergence criterion is set to $10^{-5}$ 
$\AA$ ). The system temperature (and pressure) in NVT and NPT simulations are monitored by means of the Langevin dynamics approach detailed in Ref. ${ }^{65}$ and of the Nosé-Hoover barostat ${ }^{66}$, respectively. Solute atom and PPP induced dipole moments are iteratively solved until the mean difference in these dipoles between two successive iterations is less than $10^{-6}$ Debye.

The center of mass of the $\mathbf{P 7 0} /$ counter ions system is regularly reset to the simulation box center (and the PPP coordinates updated accordingly) along our MD simulations. However to prevent any solute atom to leave that box we also consider the repulsive potential $U_{\text {box }}^{r e p}$ acting only at the simulation box boundaries in order to restrain the solute atoms within the simulation box core. Let us consider a solute atom whose cartesian coordinates are $(x, y, z)$ and a simulation box whose dimensions are $\left(L_{x}, L_{y}, L_{z}\right), U_{\text {box }}^{r e p}$ is defined as

$$
U_{\mathrm{box}}^{r e p}=\sum_{\xi=x, y, z} k\left[\max \left(2|\xi|-\left(L_{\xi}-\delta L\right), 0\right)\right]^{2},
$$

here $k$ and $\delta L$ are two constant parameters that are set for the present study to 5 kcal $\mathrm{mol}^{-1} \AA^{-2}$ and $3 \AA$, respectively. $U_{\text {box }}^{r e p}$ and all its derivatives vanish for solute atoms located within the simulation box and farther than $\delta L$ from its boundaries. Because of the dimensions of our cubic boxes (at least $90 \AA$ ) the effect of that potential is very weak (to not say negligible) on the P70 and counter ions dynamic.

\section{Results and discussion}

\subsection{The collapse of P70 in the SRP medium}

\subsubsection{Simulation starting structure}

The simulation Linear Starting Structure, LSS, of P70 is shown in Fig. 2. The P70 total electrostatic charge is neutralized by adding $70 \mathrm{Cl}^{-}$counter ions that are successively set on the nodes of a cubic grid in which the LSS is set (the grid node 
dimension is $3 \AA$ ). A counter ion is set on a node (1) to minimize the Coulombic interaction energy between it and P70 and all the already added counter ions, and (2) so that the shortest distance between it and any P70 non hydrogen atom is larger than 3.5 $\AA$. The resulting $\mathbf{P 7 0 / c o u n t e r ~ i o n s ~ s t r u c t u r e ~ i s ~ t h e n ~ s e t ~ i n ~ a ~ c u b i c ~}$ box fulfilled with PPPs that are set on all the nodes of the above grid that are distant by more than $3 \AA$ from any non-hydrogen/counter ion atom. To solvate P70 in its LSS conformation, a large cubic box whose volume is $301^{3} \AA^{3}$ and comprising about 0.9M PPPs is needed to ensure that there is at least a distance of $12 \AA$ between any solute atom and the box boundaries, see Fig. 2.

\subsubsection{P70 structural evolution}

We performed 8 independent MD simulations at the 2 ns scale of the $\mathbf{P} 70$ /counter ions system in the NPT ensemble as dissolved in the SRP medium. Each simulation corresponds to a different set of starting atom/particle velocities randomly set and corresponding to a temperature of $300 \mathrm{~K}$. Along the first $200 \mathrm{ps}$ simulation segments, we constrained P70 to its LSS using a harmonic potential restraining the position of its nitrogens. That potential was then removed. We plot in Fig. 4 the temporal evolution of the polymer gyration radius $R_{g}$ and of the ratio $R^{\text {inertia }}$ between the largest and smallest P70 inertia moment values.

As soon as the harmonic potential preventing polymer structural transitions is removed, $R^{\text {inertia }}$ evolves to reach a value included within 1 and 2 and $R_{g}$ converges towards a value of $14 \pm 0.2 \AA$ within at most $1.2 \mathrm{~ns}$, regardless of the simulation. P70 collapses thus rapidly towards a Compact Quasi Spherical, CQS, conformation, see Fig. 2. Note that we performed also these simulations however in the NVT ensemble (see our protocol detailed in Section 2.4) from a starting structure relaxed by performing a NPT MD run along which the linear P70 structure is harmonically constrained. Along these NVT trajectories, P70 collapses again towards a CQS conformation but usually at a slower rate (at least not before $2 \mathrm{~ns}$ ). To our opinion 
that difference in the collapsing time scales arises from the thermostat and barostat that we consider. That may also explain the much longer time needed by P70 to collapse along our LRP simulations that are performed in the NVT ensemble (see below).

The P70 compact conformations at the end of each simulation differ at the atomic level. For instance the Root Mean Square Deviation, RMSD, of the ammonium nitrogens $\mathrm{N}_{\mathrm{a}}$, among the 8 final simulation snapshots amounts to $7 \AA$. However the structural properties of the ammonium groups within these final conformations are close on average. In Fig. 4 we plot the radial $\mathrm{N}_{\mathrm{a}} / \mathrm{N}_{\mathrm{a}}$ pair distribution functions $g_{\mathrm{NN}}$ as computed along the last $500 \mathrm{ps}$ simulation segments as well as the mean numbers $N_{\mathrm{N}_{\mathrm{a}}}(r)$ of $\mathrm{N}_{\mathrm{a}}$ atoms lying within a sphere of radius $r$ from the Polymer Center of Mass PCOM (i.e. the integrals of $\mathrm{N}_{\mathrm{a}} / \mathrm{PCOM}$ radial distribution functions). These plots show all the $\mathrm{N}_{\mathrm{a}}$ conformations to be very close at the end of each independent simulation. Hence P70 evolves towards different compact conformations whose cationic charge distribution (that governs the electrostatic interactions within the polymer and between the polymer and its chemical environment) are close, suggesting these conformations to be iso-energetic in the SRP medium.

Regarding counter ions, we plot in Fig. 4 their mean radial distribution functions $g_{\mathrm{ci}}$ from PCOM, computed from the last 500 ps simulation segments, as well as their integrals yielding the mean number of counter ions $N_{\mathrm{ci}}$ within a sphere of radius $r$ from PCOM. The functions $g_{\mathrm{ci}}$ are close : they present two peaks located at 15 and $23 \pm 1 \AA$ from PCOM and encompassing 5 and $68 \pm 1$ counter ions, respectively. About $8 \%$ of the counter ions are thus absorbed on the $\mathbf{P} 70$ surface whereas $90 \%$ of them are located in a more distant shell extending from 4 to $21 \AA$ from the polymer surface. Lastly, at most 2 counter ions are present within the polymer CQS core and only one is released far away from P70 along a single simulation. Counter ions were distributed all along the P70 LSS. The counter ions undergo relatively large displacements at the early stages of our simulations before to form a spherical 
cloud. In the starting linear structure the counter ions mainly interact only with a few cationic charges of $\mathbf{P} 70$ whereas they interact all with all the $\mathbf{P} 70$ cationic charges located in rather small spherical volume at the end of the simulations.

In Fig. 4 we also plot the radial distribution functions $g_{\mathrm{PPP}}(r)$ between the PPPs and PCOM as well as their integrals $N_{\mathrm{PPP}}(r)$. The functions $g_{s}(r)$ are converged to their expected bulk value for $\mathrm{PPP} / \mathrm{PCOM}$ distances $r \leq 30 \AA$, i.e. at about $16 \AA$ from the P70 surface. We note also (1) a depletion of the PPP density at the vicinity of the polymer surface, that may be interpreted as resulting from the hydrophobic nature of P70, and (2) by a PPP density reinforcement in between the P70 surface and the external counter ions shell. Lastly a far from negligible number of PPPs (about 270) are trapped within the core of the P70 CQS conformations: their density within the P70 spherical volume is 0.024 PPPs per $\AA^{3}$, a value $40 \%$ weaker than within the solvent bulk.

To assess the stability of a P70 CQS conformation on longer simulation times, we select a final CQS structure (together with its counter ion cloud) from one the above independent simulations. That structure is embedded in a new cubic box whose volume is $90^{3} \AA^{3}$ and comprising $23 \mathrm{k}$ PPPs. We then performed a 500 ns scale NPT MD simulation of that solvated system. Along the new simulation, P70 jumps from its starting structure towards more and more different ones (the $\operatorname{RMSD}\left(\mathrm{N}_{\mathrm{a}}\right)$ value increases up to $5 \AA$ ) but the new structures all correspond to CQS conformations, see the superimposition of P70 structures in Fig. 5. Along that 500 ns simulation, the counter ion cloud and solvent structural properties all agree with those discussed above. Hence, in a salt free SRP medium and at infinite dilution conditions, the P70 structure fluctuates between different CQS conformations whose properties are close on time intervals close to the $\mu$ s scale. However we can not conclude from our simulations on the thermodynamical nature (global minima of the potential energy surface or transient structures ${ }^{67}$ ) of these CQS conformations. 


\subsubsection{Energies}

To discuss the energetic data, we performed a single 1 ns NPT simulation of P70 constrained in its LSS conformation and embedded together with its counter ion cloud within a $0.9 \mathrm{M}$ first order PPP cubic box. The mean values of all the interaction energy components computed from the last $800 \mathrm{ps}$ segment of that simulation are taken as the reference energy values at the beginning of the HPE collapse process.

In Fig. 6, we plot the temporal evolutions of the total intra-P70 and intra-counter ions energies, of the P70/PPPs and counter ions/PPPs polarization energies, and of the total P70/counter ions interaction energy as soon as the polymer constraints are removed along our independent simulations. The dispersion in these energy values among the simulations is weak (at most $1 \%$ ) showing all the final $\mathbf{P 7 0} /$ counter ions structures to be close in energy, as expected from the similarity of the P70 charge distribution at the end of all the simulations. Because of the large P70 electrostatic positive charge $(+70 \mathrm{e})$ and thus from the large negative charge of the counter ion cloud, there is a large increase in both the intra polymer $\left(\Delta \bar{U}_{\mathrm{p}}\right)$ and intra counter ions $\left(\Delta \bar{U}_{\mathrm{ci}}\right)$ energies during the polymer collapse process : $\Delta \bar{U}_{\mathrm{p}}$ and $\Delta \bar{U}_{\text {ci }}$ amount to about +25 and $+1510^{3} \mathrm{kcal} \mathrm{mol}^{-1}$, respectively. However these large energy increases are largely counter balanced by strong stabilizing P70 and counter ions polarization interactions with the solvent, as well as by strong stabilizing P70/counter ions interactions : during the polymer collapse process, the decreases $\Delta \bar{U}_{\mathrm{ps}}, \Delta \bar{U}_{\mathrm{cs}}$ and $\Delta \bar{U}_{\mathrm{pc}}$ in the energies corresponding to the latter interactions amount to $-5,-16$ and $-3510^{3} \mathrm{kcal} \mathrm{mol}^{-1}$, respectively.

Within our hybrid modeling framework, the collapse of P70 towards CQS conformations in a SRP medium is driven by a competition between (1) strong destabilizing intra-P70 and intra-counter ion cloud interactions and (2) strong stabilizing P70/solvent and P70/counter ions ones. By analyzing in details the main components of the interaction energies (namely the repulsion, Coulombic, polarization and dispersion components), interactions among counter ions are largely dominated by 
repulsive Coulombic effects. Intra-P70 interactions are also dominated by repulsive Coulombic effects and by far from negligible attractive polarization interactions that favor P70 CQS conformations : the variation of the intra-P70 polarization energy during the collapse process amounts to about $20 \%$ of the Coulombic one in absolute values. We may also note that intra-P70 dispersion and atomic repulsion equally favor/disfavor the CQS conformations, however the corresponding energy variations during the collapse process are one order of magnitude smaller than for polarization. Lastly we identified no more than 7 intra-P70 hydrogen bonds among the acrylamide groups along the simulations. That corresponds to an interaction energy of about $35 \mathrm{kcal} \mathrm{mol}^{-1}$, which is negligible compared to the main intra-P70 Coulombic and polarization energy components.

The attractive polarization forces within the P70 CQS conformations are tied to strong alkyl carbon induced dipole moment values $\mu_{\mathrm{C}}$ that can reach values as large as 5 Debye. In Fig. 7 , we plot the mean values of $\mu_{\mathrm{C}}$ for each carbon computed along all the final $500 \mathrm{ps}$ simulation segments as a function of the mean distance of the alkyl carbons from PCOM. In line with the expected intensity of electric fields generated by quasi spherical positive charge distributions, the values $\mu_{\mathrm{C}}$ increase as the alkyl carbons are closer to the surface of P70 in a CQS conformation. Regarding the strong $\mu_{\mathrm{C}}$ values, we may note here that strong induced dipole moments (larger than 2 Debye) are also predicted for water lying at the vicinity of the heavy cation $\mathrm{Th}(\mathrm{IV})$ or the halide anion $\mathrm{F}^{-}$in liquid water by both polarizable all-atom force field and quantum Car-Parinello simulations ${ }^{68-70}$

Contrary to the present results, a large charge fraction $f_{c}$ of 0.7 , as for $\mathbf{P} 70$, is experimentally shown to prevent a HPE to collapse towards a compact globular conformation and to favor instead elongated structures from pear-necklace to wormlike ones in different environments like water and water/acetone mixtures ${ }^{6,9-11}$. First a non negligible fraction of the counter ions (about $8 \%$ ) are adsorbed on the P70 surface. That discharging effect yields thus a slightly weaker apparent HPE $\tilde{f}_{c}$ value 
(about 0.65) than $f_{c}$. Moreover the intra-molecular stabilizing polarization effects within a P70 CQS conformation counter balance about $20 \%$ of the destabilizing intra-polymer Coulombic interactions. We may also interpret these polarization effects to be responsible for a further decrease of $\tilde{f}_{c}$ to about 0.5 . Lastly note also (1) the ionic charge to be more diluted in quaternary ammonium groups than in the $-\mathrm{NH}_{3}^{+}$and $-\mathrm{SO}_{3}^{-}$ionic heads that were used to investigate the structural properties of HPEs as a function of $f_{c}$ in Refs. ${ }^{6,9-11}$; and (2) the inter ionic head distances between adjacent polymer units in the highly charged poly(vinyl amine) HPEs of Ref. ${ }^{6}$ are about twice shorter than in P70 (see the $g_{\mathrm{NN}}$ data in Fig. 4 for instance). That yields a still weaker $\tilde{f}_{c}$ value and that explains why a HPE with a large $f_{c}$ value like P70 can collapse towards a CQS conformation in a SRP medium.

\subsubsection{Comparison to carboxylated polystyrenes PSC $^{f_{c}}$}

To further discuss the reliability of our multi level simulation scheme, we investigated the behavior of HPEs corresponding to carboxylated polystyrenes $\mathbf{P S C}^{f_{c}}$ as dissolved in first order PPP boxes. Here $f_{c}$ is the degree of carboxylation/charge fraction that we set for the present purpose to $0.00,0.25,0.50$ and 1.00 , respectively. The length of the $\mathbf{P S C}^{f_{c}}$ is 80 and their negative charge is neutralized by adding $\mathrm{Na}^{+}$counter ions. For our purpose we consider all the carboxylates as deprotonated anionic heads that are regularly spaced along the $\mathbf{P S C}^{f_{c}}$ chains. Our $\mathbf{P S C} \mathbf{f}^{f_{c}}$ are similar to the sulfonated polystyrenes experimentally investigated by Boué and co workers ${ }^{11}$ even if their length is about one order of magnitude shorter. Note the distance between two adjacent ionic heads in the linear and fully carboxylated $\mathbf{P S C}$ is about $2.5 \AA$ whereas it is at least twice as large in any P70 CQS conformation.

To fully solvate the linear starting structures of the $\mathbf{P S C}^{f_{c}}$ we need smaller PPP boxes as compared to P70 : their dimensions are now about $200 \AA$ and they comprise about 260k first order PPPs. For each solvated $\mathbf{P S C}^{f_{c}}$ we performed a single MD simulation at the $100 \mathrm{~ns}$ scale according to our MD protocol detailed 
above. As for $\mathbf{P} 70$ all the force field parameters to model intra $\mathbf{P S C} \mathbf{f}^{f_{c}}$ and $\mathbf{P} \mathbf{S C}^{f_{c}}$ / counter ion interactions are assigned only from quantum ab initio computations and taken from Ref. ${ }^{31}$.

The temporal evolutions of the gyration radii $R_{g}$ and the final snapshots of our $\mathbf{P S C}^{f_{c}}$ are provided as Supplementary Material. Contrary to P70 the fully carboxylated $\mathrm{PSC}^{1}$ does not collapse towards a compact conformation along the $100 \mathrm{~ns}$ trajectory: rather it slowly evolves towards a helicoidal structure stabilized by salt bridges between carboxylate heads and $\mathrm{Na}^{+}$ions. The three other $\mathbf{P S C} \mathbf{f}^{f_{c}}$ collapse towards more and more compact (and less organized) conformations. In particular PSC $^{0}$ collapse fastly (within a few ns) towards a stable compact conformation close to the P70 CQS ones. Moreover the $\mathbf{P S C}^{f_{c}}$ gyration radii decrease as $f_{c}$ decreases, from $22\left(f_{c}=1\right)$ down to $11\left(f_{c}=0\right) \AA$. Even if Boué and co workers ${ }^{11}$ experimentally investigated longer sulfonated polystyrene chains at $0.1-0.3 \mathrm{M}$ concentrations, our simulation results regarding our $\mathbf{P S C} \mathbf{f}^{f_{c}}$ are in line with their experimental conclusion ${ }^{11,54}$ : the $\mathbf{P S C}^{f_{c}}$ gyration radius $R_{g}$ depends increasingly on $f_{c}$.

In all our P70 and PSC $^{f_{c}}$ simulations clearly show the variability of HPE behaviors in water, behaviors that can not be modeled by considering only the HPE charge fraction $f_{c}$. In the particular case of P70, we will discuss below another pivotal parameter to understand HPE behaviors, i.e. the polarizability of the neutral HPE moieties.

\subsection{The collapsed conformations of P70 in the LRP medium}

We performed a new set of 8 independent NVT simulations at the 10 ns scale of $\mathbf{P 7 0}$ surrounded by its counter ion cloud in the LRP medium. The simulation starting structures correspond the final ones of the SRP simulations discussed above : the first level box comprising P70 in a CQS conformation, the counter ions and the 0.9M first level PPPs is embedded in a second level cubic box whose volume (about $216 \mathrm{~nm}^{3}$ ) is 8 times larger than the relaxed volume of the first level PPP box and 
that is fulfilled by about $0.9 \mathrm{M}$ second level PPPs. The molecular size of that new

LRP system is thus equivalent to a $8 \mathrm{M}$ water molecules system.

\subsubsection{Structural properties}

In Fig. 8 we plot the temporal evolution of the $\mathbf{P} 70$ gyration radius $R_{g}$ along the new simulations. These plots show a non-negligible and systematic contraction of $R_{g}$ along these simulations from 14.1 (SRP) to $12.4(\mathbf{L R P}) \pm 0.1 \AA$. In LRP, the P70 CQS conformations are thus more compact than in SRP, by about $20 \%$ in volume. However, the most striking difference between SRP and LRP simulations concerns the counter ion cloud. In Fig. 8 we plot the functions $g_{\mathrm{ci}}$ and $N_{\mathrm{ci}}(r)$ computed along the last $2 n s$ LRP simulation segments. If about $10 \%$ of the counter ions are adsorbed on the polymer surface as in SRP, only $24 \%$ of them now form a first shell lying at the vicinity of the polymer surface (as in the SRP case) and $66 \%$ of them form a second shell away from P70 (it is centered at about $60 \AA$ from PCOM).

Regarding first order PPPs, their structural features at the vicinity of the P70 surface are close to the SRP case, as well as their number trapped within the $\mathbf{P 7 0}$ CQS conformations (again about 270, see Fig. 4). In all the P70 CQS conformations are stable in LRP. However they are more contracted and their counter ion cloud is strongly altered compared to $\mathbf{S R P}$, with a large fraction of the counter ions lying now away from P70.

\subsubsection{Energies}

The energy components $\Delta \bar{U}_{\mathrm{p}}, \Delta \bar{U}_{\mathrm{ci}}, \Delta \bar{U}_{\mathrm{pc}}, \Delta \bar{U}_{\mathrm{ps}}$ and $\Delta \bar{U}_{\mathrm{cs}}$ computed along the last 2 ns segments of the LRP simulations are summarized in Tab. 1. As expected the largest differences between SRP and LRP data arise from interactions involving the counter ions and the PPPs. Because of the release of the counter ions away from P70, the magnitude of the P70/counter ions interaction energy $\Delta \bar{U}_{\mathrm{pc}}$ and of the intra counter ion cloud energy $\Delta \bar{U}_{\text {ci }}$ decreases from SRP to LRP by about $90 \%$ 
and $80 \%$, respectively. On the other hand long range solute/solvent interactions strongly reinforce the stability of the polymer CQS conformations : the magnitudes of $\Delta \bar{U}_{\mathrm{ps}}$ and $\Delta \bar{U}_{\mathrm{cs}}$ are from 3 to 4 times stronger along LRP simulations compared to SRP ones, and that arises mainly from the second level PPPs.

From Eq. 7, we may estimate the order of magnitude of the hydration long range electrostatic free energy $\Delta G_{\mathrm{lr}}$ of a solute of charge $Q$ (modeled as a point charge and whose gyration radius is $R_{g}$ ) dissolved in a homogeneous second level PPPs shell extending from $R_{\text {cut }}^{\mathrm{pol}, 1}+R_{g}$ to $R_{\mathrm{cut}}^{\mathrm{pol}, 2}$ according to

$$
\Delta G_{\mathrm{lr}}=-\frac{\alpha_{s, 2}}{2} \frac{Q^{2}}{4 \pi \epsilon_{0}} \int_{R_{\mathrm{cut}}^{\mathrm{pol}, 1}+R_{g}}^{R_{\mathrm{cut}}^{\mathrm{pol}, 2}} \frac{\rho_{s}}{R^{4}} 4 \pi R^{2} d R=-\frac{Q^{2}}{8 \pi \epsilon_{0}}\left(\frac{1}{R_{\mathrm{cut}}^{\mathrm{pol}, 1}+R_{g}}-\frac{1}{R_{\mathrm{cut}}^{\mathrm{pol}, 2}}\right)
$$

For P70 in a SRP CQS conformation, that yields $\Delta G_{\mathrm{lr}} \approx-2610^{3} \mathrm{kcal} \mathrm{mol}^{-1}$, a value in line with the $\Delta \bar{U}_{\mathrm{ps}}$ data of Tab. 1 . That relation can be readily rewritten to estimate the hydration short range solute/solvent electrostatic free energy $\Delta G_{\mathrm{sr}}$ (arising from first level PPPs) as

$$
\Delta G_{\mathrm{sr}}=-\frac{Q^{2}}{8 \pi \epsilon_{0}}\left(\frac{1}{R_{g}}-\frac{1}{R_{\mathrm{cut}}^{\mathrm{pol}, 1}+R_{g}}\right) .
$$

These relations suggest that the more compact is a HPE CQS conformation the stronger it is stabilized by both short and long range HPE/solvent electrostatic interactions. For instance the gyration radius $R_{g}$ smaller by $1.7 \AA$ in LRP than in SRP is responsible for an overall large decrease of $\Delta G_{\mathrm{sr}}$ by about $-5700 \mathrm{kcal}$ $\mathrm{mol}^{-1}$ according to the above relation. Within the $\mathbf{P} \mathbf{P} \mathbf{P}^{l}$ framework, long range P70/solvent electrostatic effects favor the contraction of a CQS conformation and thus the increase of the local charge densities within P70, a phenomenon which in turn reinforces both the short and long range solvent/polymer electrostatic interactions. However the contraction of a CQS conformation also yields to strengthen both the intra HPE repulsive Coulombic and attractive polarization effects, by about 25 
and $40 \%$, respectively (see Tab. 1).

We assess the stability of one of the final LRP CQS conformations by performing a 500 ns simulation of it embedded together with its counter ion cloud in a first level cubic box whose volume is $21.9 \mathrm{~nm}^{3}$ (and comprising about 80k first level PPPs), and in a 8 times larger second level box (comprising also about 80k second level PPPs). In that particular case $R_{\mathrm{cut}}^{\mathrm{pol}, 2}$ is $62.5 \AA$. We observe here also the $\mathbf{P} 70$ structure to jump among different CQS conformations whose structural and energetic properties are close. In particular, their gyration radii are smaller than in SRP by about 1.7 $\AA$, on average, see Fig. 5.

\subsubsection{Second order PPPs and the distant counter ion shell}

The P70 PCOM/second order PPP radial distribution functions reported in Fig. 8 (d) show over concentration of second order PPPs in between P70 and the distant counter ion shell, a domain in which the electric field components generated by opposite charged solutes are expected to be strong (whereas they mostly cancel each other for like charged solutes, see Fig. 1 and Fig. 3-B). Hence the organized P70/second order PPPs/counter ions shell structures observed at the end of our LRP simulations yields to reinforce the polarization interactions among the second order PPPs, P70 and a set of counter ions. In our original study, we already showed the density of second order PPPs to be slightly increased at the lower boundary of their cut off shell domain ${ }^{36}$ as they interact with a single monovalent ion like $\mathrm{Cl}^{-}$ or $\mathrm{Na}^{+}$. Here the large electrostatic charge of P70 (and of its counter ions cloud) yields to overestimate that phenomenon. In Section 4 we show how the decrease its intensity by reinforcing the repulsion of second order PPPs at short range. However even in that case the above conclusion regarding the stability and the contraction (and its magnitude) of the P70 CQS conformations arising from long range solvent effects remains valid. 


\subsubsection{Comparison to carboxylated polystyrene PSC PSc $^{f_{c}}$}

We also simulated the carboxylated $\mathbf{P S C} \mathbf{S}^{0.5}$ and $\mathbf{P S C}{ }^{1}$ in the $\mathbf{L R P}$ medium. The linear $\mathbf{P S C}^{f_{c}}$ starting structures (comprising $\mathrm{Na}^{+}$counter ions) correspond to those embedded in a 260k first order PPP box as detailed above. These hydrated structures are here embedded in second order PPP boxes comprising also about 260k particles. For each $\mathbf{P S C}^{f_{c}}$ we performed a single $100 \mathrm{~ns}$ MD simulations. Anticipating the conclusions of Section 4 , we set the parameter $\epsilon_{2}$ to $1 \mathrm{kcal} \mathrm{mol}^{-1}$. The temporal evolutions of the gyration radii and the final snapshots at the end of these simulations for both the $\mathbf{P S C}^{f_{c}}$ are provided as Supplementary Materials.

In LRP, the final conformation of $\mathbf{P S C}^{1}$ is more elongated than in SRP: its gyration radius $R_{g}$ is $31 \AA$ in $\mathbf{L R P}$, larger by $41 \%$ than in SRP. A weaker fraction of counter ions $\mathrm{Na}^{+}$interact at short range from carboxylate heads in LRP than in SRP: as stated in Ref. ${ }^{54}$ that yields to strengthen the intra HPE destabilizing forces arising from the Coulombic interactions among ionic heads and that explains the more elongated conformation of $\mathbf{P S C}{ }^{1}$ in $\mathbf{L R P}$ than in SRP. Regarding $\mathbf{P S C} \mathbf{C}^{0.5}$ its final conformation in $\mathbf{L R P}$ is also more elongated than in $\mathbf{S R P}\left(R_{g}=26 \AA\right)$ and we note the formation of two "pearls" at both its extremities and whose cores are stabilized by clusters of neutral phenyl groups. Such a conformation is in line with the interpretation of experimental data provided in Ref. ${ }^{54}$. As for $\mathbf{P S C}^{1}$ a weaker fraction of counter ions interact at short range from carboxylate heads in the final conformation of $\mathbf{P S C}^{0.5}$ in LRP than in SRP. That also favors its more elongated conformation in LRP.

Hence our simulations show long range solvent effects to favor different kind of HPE conformations : for $\mathbf{P 7 0}$ they reinforce the stability of its CQS conformations whereas for $\mathbf{P S C}^{f_{c}}$ with a high charge fraction $\left(f_{c} \geq 0.5\right)$ they favor elongated conformations. Hence our multi level PPP approach does not systematically favor CQS-like conformations for highly charged HPEs. 


\subsection{Intra-polymer polarization}

\subsubsection{SRP medium}

To further investigate the role of intra molecular polarization on $\mathbf{P 7 0}$ conformations solvated in a salt free SRP medium at infinite dilution conditions, we performed two sets of 8 independent NVT simulations at the $10 n s$ scale of the $\mathbf{P 7 0} /$ counter ions system embedded in a $0.9 \mathrm{M}$ first order PPPs cubic box, however by setting to zero all the atomic polarizabilities. For these two simulation sets, their starting structures correspond to those obtained at the end

1. of the initial 200 ps segments of the polarizable $\mathbf{P} 70$ simulations in $\mathbf{S R P}$, segments along which P70 is harmonically constrained in its LSS conformation;

2. of the polarizable $\mathbf{P} 70$ simulations in $\mathbf{S R P}$, i.e. $\mathbf{P} 70$ is in a CQS conformation.

These starting structures are relaxed by performing preliminary 200 ps NVT simulations along which the $\mathbf{P} 70$ structure is constrained and corresponding to different starting velocity sets. The simulations are then restarted by removing the constraints on P70.

In Fig. 9 we plot the temporal evolutions of the $\mathbf{P} 70$ gyration radius $R_{g}$ and ratio $R^{\text {inertia }}$ along both sets of simulations. As shown by these plots, the P70 structures converge within less than $8 n s$ towards quasi spherical conformations whose $R_{g}$ value is almost equal, about $21.5 \pm 1.0 \AA$, regardless of the simulation starting structure. These final conformations, denoted as $\mathrm{QS}^{\text {nopol }}$, all present relatively large cavities within them. Compared to the CQS conformations of polarizable P70 in SRP, they are less compact by a factor 3 in volume, and they correspond more to coil/helicoidal conformations than to compact globular ones, see Fig. 2.

In Fig. 9 we also plot the P70 $g_{\mathrm{NN}}(r)$ and $N_{\mathrm{N}_{\mathrm{a}}}(r)$, the counter ion $g_{\mathrm{ci}}(r)$ and $N_{\mathrm{ci}}(r)$, and the solvent $g_{\mathrm{PPP}}(r)$ and $N_{\mathrm{PPP}}(r)$ functions computed along the last $2 n s$ segments of the non-polarizable simulations. Regardless of the simulation starting 
structure, the main features of the $g_{\mathrm{NN}}(r)$ and $N_{\mathrm{Na}_{\mathrm{a}}}(r)$ functions are close. The cationic charge distributions within all the $\mathrm{QS}^{\text {nopol }}$ conformations are thus close, suggesting their main energetic properties to be also close (see below). We may also note that, in line with the larger gyration radius of the $\mathrm{QS}{ }^{\text {nopol }}$ conformations, the present functions $g_{\mathrm{NN}}(r)$ start to be non zero for inter nitrogen $\mathrm{N}_{\mathrm{a}}$ distances $r$ larger by about $1.5 \AA$ compared to their polarizable CQS counter parts, and they vanish for distances $r$ larger than $45 \AA$, i.e. $10 \AA$ farther than for the polarizable CQSs.

The counter ion $g_{\mathrm{ci}}(r)$ and $N_{\mathrm{ci}}(r)$ functions are also close, regardless of the starting simulation structure. Contrary to the SRP simulations of polarizable P70, a large fraction of the counter ions (about $20 \%$ ) is now adsorbed within the polymer core and the remaining ones form a spherical shell centered at about $28 \AA$ from PCOM and extending up to about $18 \AA$ from the P70 'surface' (i.e. the surface of a sphere whose radius is $\left.R_{g}\right)$. Lastly, the solvent functions $g_{\mathrm{PPP}}(r)$ show the first level PPP density to be almost constant and to already reach $90 \%$ of its bulk value within the P70 core domain. That yields the hydration index (defined as the mean number of first order PPPs lying within the $\mathbf{P} 70$ core) of the $\mathrm{QS}^{\text {nopol }}$ conformations to be 4 times larger than for CQS conformations (about 1200 and 270, respectively). Hence the large cavities within the $\mathrm{QS}^{\text {nopol }}$ conformations are full filled by counter ions and first level PPPs.

As expected from the close charge distributions within the $\mathrm{QS}^{\text {nopol }}$ conformations and from the close counter ion and PPP structural properties, there are weak dispersions among the values of the mean energy components $\Delta \bar{U}_{\mathrm{p}}, \Delta \bar{U}_{\mathrm{ci}}, \Delta \bar{U}_{\mathrm{pc}}, \Delta \bar{U}_{\mathrm{ps}}$ and $\Delta \bar{U}_{\mathrm{cs}}$ computed along all the final 2 ns non-polarizable simulation segments. Compared to polarizable data, the less compact $\mathrm{QS}^{\text {nopol }}$ conformations are tied to twice weaker intra-P70 Coulombic and P70/PPPs interaction energies, see Tab. 1. According to Eqs. (9-10), the latter results may be interpreted as arising from the more diluted cationic charge within the less compact $\mathrm{QS}^{\text {nopol }}$ conformations. Interestingly the $\mathrm{QS}^{\text {nopol }}$ magnitudes of the mean intra counter ion cloud $\Delta \bar{U}_{\mathrm{ci}}, \mathbf{P} 70 /$ counter ions 
$\Delta \bar{U}_{\mathrm{pc}}$ and counter ions/solvent energies $\Delta \bar{U}_{\mathrm{cs}}$ agree with the CQS one.

\subsubsection{LRP medium}

We performed a new set of 8 independent NVT simulations in LRP whose starting P70/counter ions structures correspond to the final ones of the non-polarizable simulations in SRP. However these structures are embedded in two $0.9 \mathrm{M}$ first and second level PPP cubic boxes and they are simulated at the $20 \mathrm{~ns}$ scale. As above all the $\mathbf{P 7 0}$ atomic polarizabilities are zeroed. The main $\mathbf{P 7 0}$, counter ions and solvent structural and energetic data, averaged over the last 2 ns simulation segments, are reported in Fig. 10 and in Tab. 1.

Accounting for second level PPPs, and thus for long range solvent electrostatic effects, yields again (1) a large fraction of the counter ions to be largely repelled away from P70 (they form again a distant shell lying at about 50-60 A from PCOM), and (2) a strong contraction of the starting $\mathrm{QS}^{\text {nopol }}$ conformations : their mean gyration radius $R_{g}$ decrease from $21.5 \pm 1 \AA$ in $\mathbf{S R P}$ towards $14.2 \pm 0.2 \AA$ in $\mathbf{L R P}$. The structural details of these new LRP compact and quasi spherical conformations, denoted as CQS ${ }^{\text {nopol }}$, are overall close to the CQS ones corresponding to polarizable P70 in SRP, see Fig. 10(b). However, regarding the counter ions, the noticeable fraction of them (about 20\%) that were trapped within the starting $\mathrm{QS}^{\text {nopol }}$ conformations are still trapped within the final CQS ${ }^{\text {nopol }}$ ones. All these results may be interpreted as above for the polarizable P70 embedded in the LRP medium. However the large fraction of the counter ions trapped within the CQS ${ }^{\text {nopol }}$ final conformations are responsible for a strong HPE discharging effect that stabilizes these conformations as intra-P70 polarization attractive forces favor CQS conformations.

As for polarizable $\mathbf{P 7 0}$, the $\mathbf{P 7 0}$ /counter ion radial distribution functions reported in Fig. 10 show here also the existence of a distant counter ion shell lying at $60 \AA$ from PCOM. As discussed for polarizable P70, the origin of that shell arises here also from over concentration of second order PPPs in between that shell and 
P70. As it will be discussed in Section 4, reinforcing the short repulsion among second order PPPs will yield that distant shell to vanish. However that will not lead to invalidate the present conclusions about the contraction of the non polarizable P70 conformations arising from long range solvent effects.

At the exception of polarization and counter ion energy data, the energetic data reported in Tab. 1 for non-polarizable $\mathbf{P 7 0}$ in LRP are in line with their polarizable P70 counter parts. In particular we note again the strong stabilization of the $\mathrm{CQS}^{\text {nopol }}$ conformations arising from long range solvent electrostatic effects. Regarding counter ions, long range solvent electrostatic effects favors their structure as for polarizable P70, but more strongly as suggested by the magnitude of the energy component $\Delta \bar{U}_{\mathrm{cs}}$. As that component only measures the strength of the counter ion/solvent interaction, its strong intensity here may result from the large fraction of counter ions trapped within the overall small volume corresponding to CQS ${ }^{\text {nopol }}$ conformations, as suggested by Eqs. 9 and 10.

Besides confirming the role of electrostatic long range solvent effects, our nonpolarizable P70 simulations clearly show the strong effect of intra-molecular polarization on the conformation of a HPE in liquid water-like SRP and LRP media. According to our modeling framework, these intra-molecular polarization effects are not tied to the strongest interaction energies within our HPE/counter ions/solvent systems, but they are a key factor favoring HPE compact globular conformations.

\section{HPE collapse and the solvent coarse grained ap- proach}

Coarse-graining the solvent yields approximations in modeling solute/solvent interactions. As even conclusions from all atom simulations regarding the association of standard ion pairs in aqueous phase vary noticeably when considering different sets of all atom force field parameters ${ }^{71-73}$, one may wonder about the reliability of 
simulations based on a solvent coarse grained approach to investigate the behavior of a heavily charged HPE like P70.

In an earlier study ${ }^{37}$, we showed the $\mathbf{P P P}^{l}$ approach to meet the predictions of the lattice numerical method implemented in the APBS code $^{74}$ to solve the Poisson Boltzman equations as computing the electrostatic component of the hydration free energy of proteins, a particular kind of polyelectrolytes. Here we further investigate the $\mathbf{P P P}^{l}$ accuracy by performing an additional set of eight simulations of $\mathbf{P} 70$ embedded in 1M first level PPP box. These simulations correspond to polarizability values $\alpha_{s}$ of the PPPs that range from 1.2 up $2.7 \AA^{3}$ (the $\alpha_{s}$ value computed from Equation (4) is $2.35 \AA^{3}$ ). Each simulation was performed as detailed above up to 10 ns from the P70 linear starting structure. In Fig. 11 we plot the mean P70 gyration radius $R_{g}$ and the mean $\mathbf{P} 70 /$ solvent interaction energy $U_{\mathrm{ps}}$ as a function of $\alpha_{s}$ (these mean quantities are computed over the final simulation segment on which the P70 structure is stable). From these plots it appears that P70 collapses within 10 ns towards a CQS conformation as soon as $\alpha_{s}=1.8 \AA^{3}$, a value $30 \%$ smaller than the one we used in the above simulations. Regarding the $U_{\mathrm{ps}}$ values, their magnitude for the P70 CQS conformation is a decreasing linear function of $\alpha_{s}$ : it ranges from $-20\left(\alpha_{s}=1.8 \AA^{3}\right)$ down to $-39\left(\alpha_{s}=2.7 \AA^{3}\right) 10^{3} \mathrm{kcal} \mathrm{mol}^{-1}$.

We simulated P70 in its CQS conformation as embedded in a spherical cavity whose radius is $43 \AA$ and comprising about 7500 explicit and polarizable water molecules. P70 and water were simulated using an all atom polarizable force field whose accuracy is discussed in Refs. ${ }^{56,75}$ (see also Supplementary Material for details). That all atom simulation yields a $U_{\mathrm{ps}}$ value that ranges from -26 to $-2810^{3}$ kcal $\mathrm{mol}^{-1}$ as accounting for water molecules lying within a shell extending up to $12 \AA$ from the P70 boundary (as defined in Section 2.3). Such a $U_{\mathrm{ps}}$ value is fully in line with our $\mathbf{P P P}^{l}$ estimate for $\alpha_{s}=2.35 \AA^{3}$.

Defining HPE structures by analogy with proteins, the secondary structure of P70 for low values $\alpha_{s}$ (up to $1.8 \AA^{3}$ ) corresponds to long helices where the ammo- 
nium groups point towards the solvent and they interact at short range with the counter ions. These helices can adopt neck-lace, wormlike or more compact folded conformations (see Supplementary Material) showing the wide range of potential P70 tertiary structures in low to moderately polarizable solvents. For instance the P70 tertiary structure along our simulations is a neck lace for $\alpha_{s}=1.2 \AA^{3}$ and wormlike for $\alpha_{s}=1.4 \AA^{3}$ (that explains their respective gyration radius values in Fig. 11). However both kind of structures are very close in energy, regardless of the latter $\alpha_{s}$ values.

For large $\alpha_{s}$ values, the helix secondary structure vanishes and P70 collapses towards more disordered, compact and quasi spherical conformations as discussed in the above section. Around these CQS conformations we note the counter ions to form a more and more diffuse cloud that extends farther and farther from the P70 boundary as $\alpha_{s}$ increases. Whereas salts were considered as fully dissociated in water at low to moderate concentrations, large salt/water aggregates are now inferred to be abundant even in dilute solutions ${ }^{76-79}$. The stability of such edifices may be explained from similar arguments as those schematized in Fig. 1(b) : the stability of polarizable water molecules intercalated between two species of opposite charges is reinforced by the resulting non zero electric field generated by the latter charges on them. These effects are responsible for the stability of the well known Solvent Separated Ion Pair, SSIP, conformations that are pivotal in the ion pair dissociation process in aqueous phase (see Ref. ${ }^{80}$ and the references mentioned therein). Our hybrid simulations show SSIP-like P70/counter ions to be more and more favored as $\alpha_{s}$ increases.

Lastly we have to take in mind that our simulations are performed in infinite dilution conditions. The HPE solutions that are experimentally investigated comprise an overall large number of HPE chains, each of them generating local electric fields on the solvent. Even if these fields undergo large fluctuations, they will on average less polarize the solvent located in between two HPE chains than the solvent lying 
at the vicinity of a single and isolated HPE chain as in the present study. That arises form the electric field cancellation effect detailed in Fig. 1(b) (that effect is thus opposite to the above one favoring SSIP-like conformations). Hence within our $\mathbf{P P P}^{l}$ frame work, increasing the concentration of HPE is expected to weaken HPE/solvent interactions and thus to destabilize HPE CQS conformations and to favor more elongated HPE ones.

Regarding second order PPPs and the magnitude of the $\epsilon_{2}$ parameter modulating the intensity of the LJ interactions among them, we performed two new series of eight MD simulations by considering P70 as a polarizable and a non polarizable entity. Instead of using a parameter $\epsilon_{2}$ one order of magnitude smaller than the one used for first level PPPs (i.e. $\epsilon_{2}=0.05 \mathrm{kcal} \mathrm{mol}^{-1}$ ), we set $\epsilon_{2}$ to a value ranging from 1 to $8 \mathrm{kcal} \mathrm{mol}^{-1}$ to perform these new simulations. Besides reinforcing the interactions among second order PPPs, these larger $\epsilon_{2}$ values also noticeably reinforce the strength of the short range repulsion among second order PPPs (see Supporting Material). That will thus prevent (at least in part) over concentration of second order PPPs at the vicinity of charged solutes. The P70 starting structure (and its counter ion cloud) correspond to one of the final CQS conformations of a polarizable simulation in SRP. That structure is embedded in a first order PPP box whose dimension is $200 \AA$. That yields a system comprising about 260k first order PPPs that is embedded in a second order PPP box comprising also about 260k particles. The simulations were performed as detailed above up to $30 \mathrm{~ns}$. The ion/P70 PCOM radial distribution functions $g_{\mathrm{ci}}(r)$ and their integrals $N_{\mathrm{ci}}(r)$ are provided as Supplementary Material. These functions show a large fraction of the counter ions to not be any more organized as a distant shell lying at about $60 \AA$ from the P70 PCOM, but rather to belong to a diffuse counter ion cloud extending within the entire simulation box. That behavior is observed for any value of $\epsilon_{2} \geq 1$ kcal mol ${ }^{-1}$. Regarding polarizable P70, its CQS conformation is stable and still more contracted as compared to simulations in $\mathbf{S R P}$ (the $\mathbf{P} 70$ gyration radii at 
the end of these new simulations are weaker by 0.6 to $1.5 \AA$ than in SRP). For non polarizable $\mathbf{P 7 0}$, its structure is also more contracted than in SRP (the P70 gyration radii range from 15 to $18 \AA$ ). For both polarizable and non polarizable P70, we do not observe a dependence of their gyration radius on the magnitude of $\epsilon_{2} \geq 1 \mathrm{kcal} \mathrm{mol}^{-1}$. Hence, even if there is room to improve the modeling of inter particle interactions for high order PPPs, all our results show solvent long range effects (as modeled by our second order PPPs) to reinforce the stability of the P70 CQS conformations and to favor more contracted CQS conformations than do only short range solvent effects.

In all the above discussions suggest our $\mathbf{P P P}^{l}$ approach to capture the main effects regarding HPE hydration. In particular our hybrid approach does not overestimate the solute/solvent interactions that stabilize CQS-like HPE conformations in the present study. It is thus a priori able to provide reliable quantitative conclusions regarding the behavior of HPEs in aqueous phase. However and contrary to our original study ${ }^{36}$ we recommend the use of large LJ $\epsilon_{k}$ parameters (i.e. $\epsilon_{k} \geq 1$ $\mathrm{kcal} \mathrm{mol}{ }^{-1}$ ) to model the interactions among PPPs whose order $k$ is higher than one.

\section{Conclusion}

We presented MD simulation results about the HPE P70 solvated in salt free liquid water-like SRP and LRP media, in presence of counter ions and at infinite dilution conditions. The SRP and LRP media are modeled by means of polarizable particles PPPs according to the multi-level CG $\mathbf{P P P}^{l}$ approach. In the SRP medium, only PPPs whose size matches that of a single water molecule are taken into account and they interact with the solutes if they lie within a shell extending to no more than $12 \AA$ from any solute non hydrogen atom. For the LRP medium, we take into account these first level PPPs as well as a new set of larger PPPs (whose volume 
is 8 times larger than that of a single water molecule) that interact only with the HPE charged groups and the counter ions if they lie within 12 to $143 \AA$ from the solutes. The two regimes behavior of the free energy corresponding to the creation of an empty cavity within liquid water (as predicted by to the Lum-Chandler-Weeks theory of hydrophobicity ${ }^{62}$ ) is reproduced by these two media. The properties of the LRP medium are close to the liquid water one ${ }^{36}$ whereas the SRP medium may be interpreted as the solvent water confined in a cavity. The HPE and its counter ion cloud are taken into account explicitly and modeled according to the all atom polarizable force field TCPEp approach ${ }^{31}$.

From a P70 linear starting structure surrounded by a thin layer of counter ions, we performed multiple simulations of that system embedded in the SRP medium modeled as a cubic box comprising about 0.9 M first level PPPs (and whose volume is $27 \mathrm{~nm}^{3}$ ). Our simulations show $\mathbf{P} 70$ to collapse in that medium towards compact and quasi-spherical conformations surrounded by a counter ion shell lying a short range (about 8-10 $\AA$ ) from the $\mathbf{P 7 0}$ surface. Even if the atomic details of these conformations differ among the simulations, the spatial distributions of their cationic groups are close suggesting these conformations to be close in energy in SRP as supported by the analysis of different energy components performed along the simulations, like intra-P70 and P70/counter ions interaction energies. The $\mathbf{P} 70$ compact and quasi spherical conformations relaxed in the SRP medium were then simulated in the LRP medium by embedding the final structures of the $\mathbf{P 7 0} /$ counter ions/0.9 M first level PPPs systems in a new cubic box comprising $0.9 \mathrm{M}$ second level PPPs and whose volume is $216 \mathrm{~nm}^{3}$. That new system accounts thus for an equivalent of $8 \mathrm{M}$ water molecules. The SRP compact and quasi spherical conformations are structurally stable in LRP, even if we note a noticeable contraction of them (by about $20 \%$ in volume) as well as a large fraction of the counter ions to be repelled at farther distances from P70 than in SRP. Both the latter results arise from long range solvent electrostatic effects that weakens the association of oppositely charged 
systems and favors the contraction of heavy charged flexible solutes.

For comparison purposes we also simulated at the 100 ns scale four carboxylated polystyrenes $\mathbf{P S C}^{f_{c}}$ (whose fraction of charge varies from 0 to 1 ) in both the SRP and the LRP medium by means of our MD protocol. These HPEs are shown by our simulations to behave as the sulfonated polystyrenes experimentally investigated by Boué and co workers ${ }^{11,54}$ : their gyration radius increases as their charge fraction increases. In particular and contrary to our P70, the fully carboxylated $\mathbf{P S C}^{1}$ evolves along a 100 ns MD trajectory towards a helicoidal structure in SRP (structure that is stabilized by local carboxylate/counter ion salt bridges) and towards a more elongated structure in LRP.

Even if the behavior of P70 in our water-like SRP and LRP media does not prejudge of the behavior of any kind of HPEs in such environments (in particular their collapse towards compact conformations as discussed for carboxylated polystyrenes PSC $^{f_{c}}$ with a high charge fraction) we may draw general conclusions from our simulations. The behavior of a HPE in any solvent is governed by a complex interplay among microscopic interactions occurring within it and between the HPE and its environment (counter ions and solvent). In particular, in salt free solutions and at infinite dilution conditions, our simulations show that

1. in line with the standard picture of the effects governing the behavior of a HPE in solution (see Ref. ${ }^{81}$ for instance), HPE compact conformations are disfavored by strong Coulombic intra HPE effects and favored by strong Coulombic $\mathrm{HPE} /$ counter ions ones, especially in a SRP medium in which the association of oppositely charged ions is more favored than in LRP;

2. solute/solvent electrostatic interactions (as described within the $\mathbf{P} \mathbf{P} \mathbf{P}^{l}$ framework) and particularly long range ones, favor HPE compact conformations : that yields the reinforcement of the HPE local density of charge which in turn strengthens the electrostatic response of a polarizable solvent like water to the 
HPE presence;

3. the existence of strong intra HPE attractive polarization effects (arising from the polarization of alkyl carbons by the HPE ionic charges, for instance) also favors HPE compact conformations.

We also simulated a non-polarizable P70 in both the SRP and LRP media. These new simulations support the key role played by intra HPE polarization effects to understand the stability of HPE compact globular conformations as in the particular case of $\mathbf{P 7 0}$. Not accounting for them in our simulations yields P70 to adopt less compact conformations : their gyration radii increase by a factor of about $50 \%$ (SRP) and 20\% (LRP) compared to the compact structures predicted by accounting for these polarization effects. In the present case, intra HPE polarization effects correspond to attractive forces. They may thus be modeled using a basic additive dispersion-like energy term. However polarization is a non-linear phenomenon that can be attractive, repulsive and even vanish for particular molecular geometries, and might be extremely relevant for studying Natural ingredients like polysaccharides as already shown for polypeptides. Hence microscopic polarization has to be explicitly taken into account not only to model HPE systems at infinite dilution conditions (as in the present study) but also in more complex and concentrated chemical environments for which it is not obvious to infer the role of polarization (like to favor/disfavor short range interactions among HPEs for instance). Our works emphasizes the importance of considering polarizability for the future of digitallyenabled formulation for Green Sciences. Lastly our hybrid modeling approach is well suited to be used in conjunction with efficient algorithms to simulate explicitly very large solutes (or mixtures of solutes), like a Fast Multipole Method devoted to induced dipole-based polarizable force fields. ${ }^{82}$ 


\section{Acknowledgments}

This work was granted access to the TGCC HPC resources under the allocation 2019-2020 [x2016081859] and the Grand Challenge allocation [GC0429] made by GENCI.

\section{Data Availability}

Data openly available in a public repository that does not issue DOIs. A full binary version of our simulation code POLARIS(MD) (version 1.2.0.1) and the input files that were used for the present study to perform simulations on a multi core processor are freely available for download ${ }^{64}$

\section{Supplementary Material}

See supplementary material for the details regarding (1) the all atom simulation of P70 embedded in an aqueous cavity and the its final conformations as a function of the solvent polarizability; (2) the simulation data of the $\mathbf{P S C}^{f_{c}}$ in the SRP and LRP media; (3) the simulation data of P70 in LRP using a LJ parameter $\epsilon_{2}$ ranging from 1 to $8 \mathrm{kcal} \mathrm{mol}^{-1}$. A short video is also provided that shows the collapse of the polyelectrolyte along a simulation in the $1 \mathrm{M}$ first level pseudo particle box.

\section{Conflicts of interest}

A. C. and F. L. are full employees of L'Oréal involved in research activities. 
Table 1: Decomposition in Coulombic $\Delta \bar{U}^{q q}$, polarization $\Delta \bar{U}^{\text {pol }}$ and non electrostatic $\Delta \bar{U}^{n e}$ energy components of the intra-P70 and intra-counter ion cloud mean energies $\Delta \bar{U}_{\mathrm{p}}$ and $\Delta \bar{U}_{\mathrm{ci}}$ and of the $\mathbf{P 7 0} /$ counter ions, $\mathbf{P} 70 / \mathrm{PPPs}$ and counter ions/PPPs interaction energies $\Delta \bar{U}_{\mathrm{pc}}, \Delta \bar{U}_{\mathrm{ps}}$ and $\Delta \bar{U}_{\mathrm{cs}}$. Data in italic correspond to the root mean square deviations of these values among the independent simulations. For LRP, the $\Delta \bar{U}_{\mathrm{ps}}$ and $\Delta \bar{U}_{\mathrm{cs}}$ data in brackets corresponds to the first level PPP contributions. The non electrostatic component $\Delta \bar{U}^{n e}$ corresponds to the energy terms $U^{\text {disp }} / U^{\text {coop }}$ or to the PPP/solute Lennard-Jones-like term (see Section 2). All values are expressed in $10^{3} \mathrm{kcal} \mathrm{mol}^{-1}$. Values smaller than $100 \mathrm{kcal} \mathrm{mol}^{-1}$ are not reported.

\begin{tabular}{|c|c|c|c|}
\hline & $\overline{\Delta \Delta U^{q q}}$ & $\overline{\Delta \Delta U^{p o l}}$ & $\overline{\Delta \Delta U^{n e}}$ \\
\hline SRP & \multicolumn{2}{|c|}{ medium and polarizable $\mathbf{P 7 0}$} & \multirow{3}{*}{$-0.30 .02-12-x$} \\
\hline$\Delta \bar{U}_{\mathrm{p}}$ & 23.90 .4 & -2.60 .1 & \\
\hline$\Delta \bar{U}_{\mathrm{ci}}$ & 9.80 .4 & -0.10 .02 & \\
\hline$\Delta \bar{U}_{\mathrm{pc}}$ & -24.10 .6 & & 0.10 .05 \\
\hline$\Delta \bar{U}_{\mathrm{ps}}$ & & $\begin{array}{lll}-11.6 & 0.3\end{array}$ & 0.10 .05 \\
\hline$\Delta \bar{U}_{\mathrm{cs}}$ & \multirow{2}{*}{\multicolumn{3}{|c|}{$\begin{array}{c}-3.2 \text { 0.1 } \\
\text { medium and polarizable } \mathbf{P 7 0}\end{array}$}} \\
\hline LRP & & & \\
\hline$\Delta \bar{U}_{\mathrm{p}}$ & 30.20 .6 & -4.60 .3 & -0.80 .1 \\
\hline$\Delta \bar{U}_{\mathrm{ci}}$ & 2.41 .3 & & 0.10 .05 \\
\hline$\Delta \bar{U}_{\mathrm{pc}}$ & -2.81 .6 & 0.30 .1 & \\
\hline$\Delta \bar{U}_{\mathrm{ps}}$ & & $-52.82\left[\begin{array}{ll}-15 & 0.3\end{array}\right]$ & 0.611 \\
\hline$\Delta \bar{U}_{\mathrm{cs}}$ & & $-13.814\left[\begin{array}{lll}-9.3 & 1.8\end{array}\right]$ & \\
\hline SRP & medium an & non polarizable $\mathbf{P} \mathbf{7 0}$ & \\
\hline$\Delta \bar{U}_{\mathrm{p}}$ & 11.90 .3 & & \\
\hline$\Delta \bar{U}_{\mathrm{ci}}$ & 10.40 .3 & & \\
\hline$\Delta \bar{U}_{\mathrm{pc}}$ & -23.80 .7 & & \\
\hline$\Delta \bar{U}_{\mathrm{ps}}$ & & $\begin{array}{ll}-5.1 & 0.2\end{array}$ & -0.1 \\
\hline$\Delta \bar{U}_{\mathrm{cs}}$ & & $\begin{array}{ll}-3.9 & 0.1\end{array}$ & \\
\hline LRP & medium an & non polarizable $\mathbf{P} 70$ & \\
\hline$\Delta \bar{U}_{\mathrm{p}}$ & 30.30 .5 & & -0.50 .1 \\
\hline$\Delta \bar{U}_{\mathrm{ci}}$ & 14.54 .8 & & \\
\hline$\Delta \bar{U}_{\mathrm{pc}}$ & -5.83 .2 & 0.10 .02 & $\begin{array}{ll}-0.1 & 0.02\end{array}$ \\
\hline$\Delta \bar{U}_{\mathrm{ps}}$ & & $\begin{array}{lll}-52.5 & 1.5\end{array}\left[\begin{array}{lll}-10.3 & 0.3\end{array}\right]$ & 0.80 .1 \\
\hline$\Delta \bar{U}_{\mathrm{cs}}$ & & $-31.87 .7[-7.71 .9]$ & 0.20 .01 \\
\hline
\end{tabular}



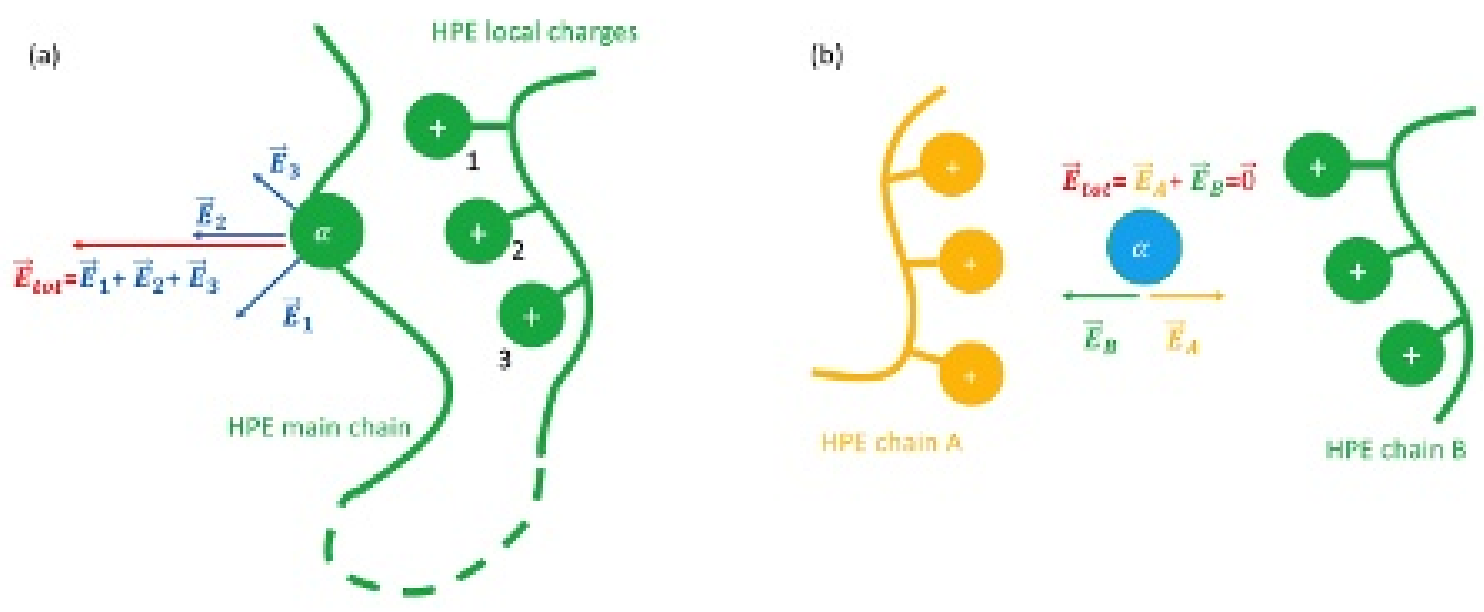

Figure 1: Microscopic polarization effects. (a) An atom, whose isotropic polarizability is $\alpha$, belonging to a HPE (in green) is located at the vicinity of three positively charged groups. All the other atoms are non-polarizable centers. That atom undergoes the total electric field $\mathbf{E}_{t o t}=\mathbf{E}_{1}+\mathbf{E}_{2}+\mathbf{E}_{3}$ arising from the three positive charges. From an induced dipole moment approach, the dipole moment generated on that atom obeys $\alpha \mathbf{E}_{\text {tot }}$ and it is at the origin of non additive polarization energy component $-\frac{1}{2} \alpha \mathbf{E}_{\text {tot }}^{2}$ that can not be modeled using standard additive potentials. (b) A solvent molecule whose polarizability is $\alpha$ is located in between two HPE chains A and B. The electric fields arising from each chain cancel out. If the charges of chain $\mathrm{A}$ are opposite to those of chain $\mathrm{B}$, then the resulting electric field on the solvent is $\mathbf{E}_{\text {tot }}=\mathbf{E}_{A}+\mathbf{E}_{B}=2 \mathbf{E}_{A}=2 \mathbf{E}_{B} \neq \mathbf{0}$, and the corresponding polarization energy on the solvent is four times greater than as arising from each single chain taken alone. 


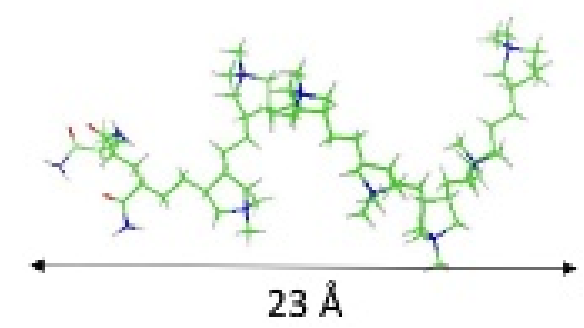

(a)
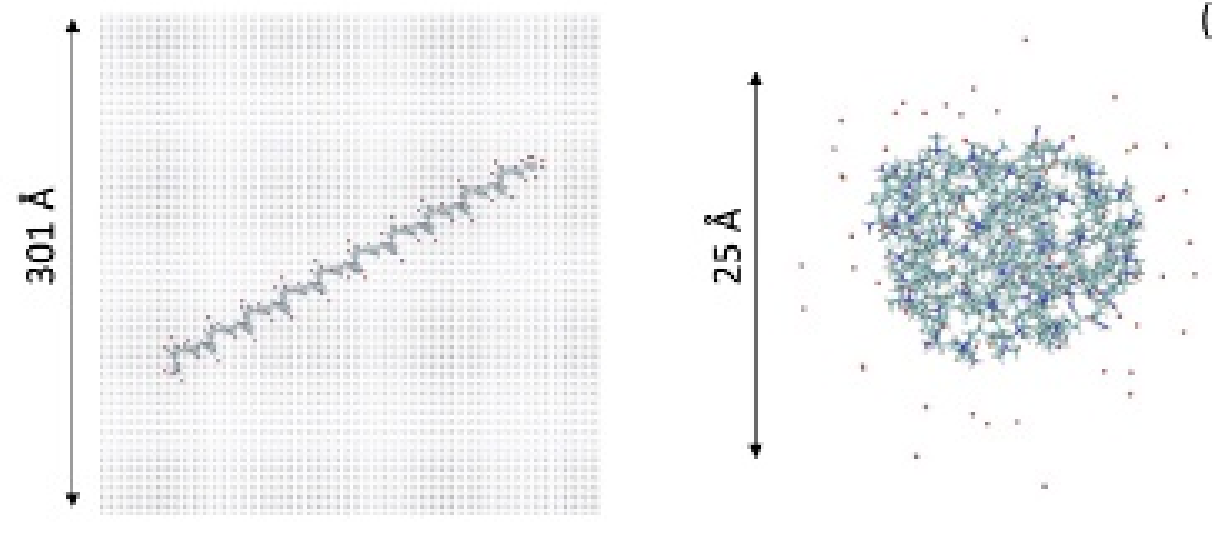

(b)
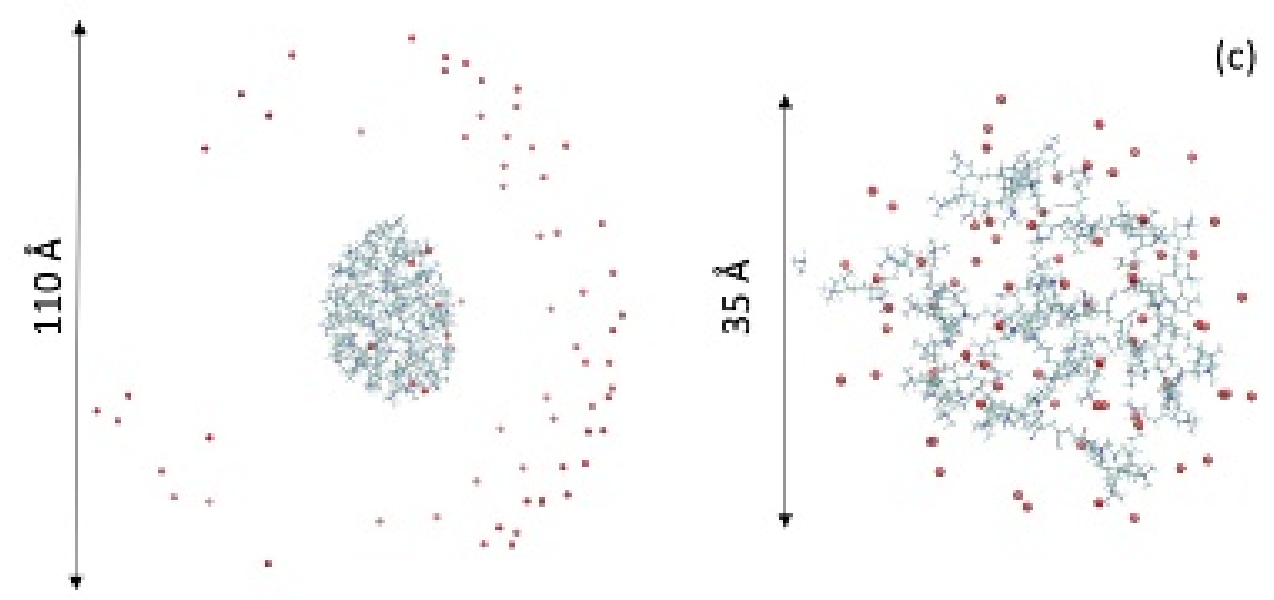

Figure 2: (a) A three dimensional representation of the main P70 unit of the modeled, generic, HPE copolymer. The HPE is made of 10 units each comprising 7 adjacent cyclo butyl ammonium cationic heads interconnect by ethyl groups, that are connected to 3 more adjacent neutral groups equivalent to a triade of asparagine proteinic residues ${ }^{38-42}$. The HPE total charge is thus $+70 e$. Here the carbon, hydrogen, nitrogen and oxygen atoms are shown in light blue, grey, dark blue and red. (b) Left : P70 in its LSS conformation embedded in a 0.9M PPPs cubic box and whose electrostatic charge is neutralized by $70 \mathrm{Cl}^{-}$counter ions (in red, PPPs are shown in blue). Right : a CQS conformation of P70 in the SRP medium. (c) Left : a CQS conformation of $\mathbf{P 7 0}$ in the LRP medium. Right : an inflated conformation of the non polarizable P70 in the SRP medium. 


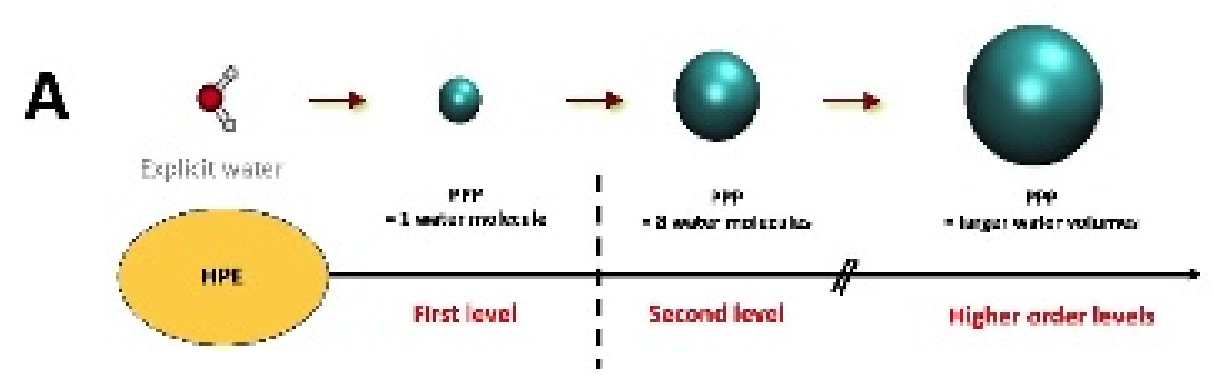

B

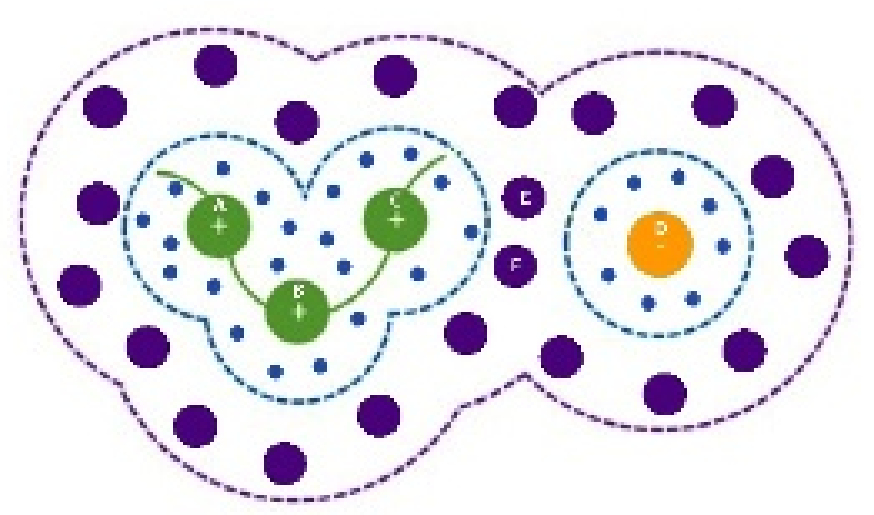

Figure 3: A The multi-level solvent CG approach $\mathbf{P P P}^{l}$. The blue spheres are PPP particles that differ by their size and thus by their polarizability. The vertical line

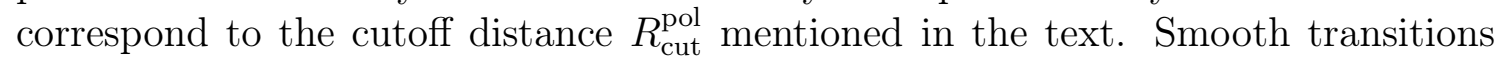
exist between the different PPP level shells, i.e. the PPP/HPE interactions are scaled by B-spline functions that smoothly vary between 1 and 0 (lower PPP level) and between 0 and 1 (higher PPP level) at solvent shell boundaries. B Example of a HPE (in green) interacting with a counter ion D (in orange) solvated by first (in blue) and second order (in violet) PPPs. The dashed lines defined the first and second order shell-based cut off domains. Any first level PPP located within the domain delimited by the blue dashed lines undergoes the total electric field generated by all the HPE and counter ion charges (denoted here as A, B, C and D). Any second level PPPs located within the domain delimited by the blue and the violet dashed lines also undergoes the latter electric field. 

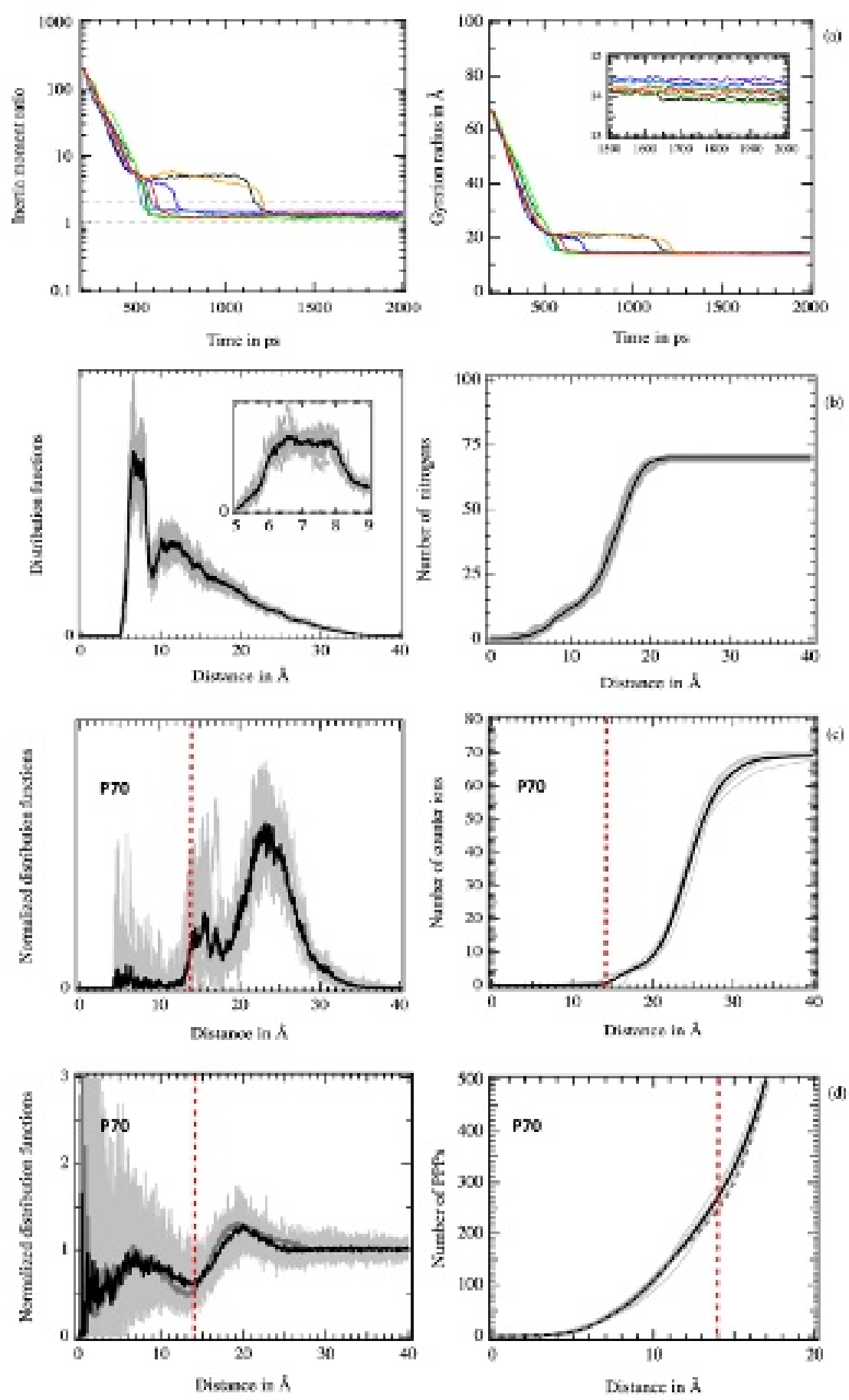

Figure 4: (a) Right : temporal evolution of the ratio between the smallest and largest inertia moment values (left) and of the gyration radius (right) of P70 along the independent simulations in the SRP medium. (b) Mean radial pair distribution function $\bar{g}_{\mathrm{NN}}$ (left, the inset shows the details of its first peak) and mean number $\bar{N}\left(\mathrm{~N}_{\mathrm{a}}\right)$ (right). (c) Mean radial distribution function $\bar{g}_{\mathrm{ci}}(r)$ (left) and its corresponding integral $N_{\mathrm{ci}}(r)$ (right). (d) Mean radial PPP/PCOM distribution function (left) and its corresponding integral (right). From (b) and (d) : the data from each independent simulation are shown by grey lines. The vertical dashed red line is located at the converged P70 mean gyration radius. 

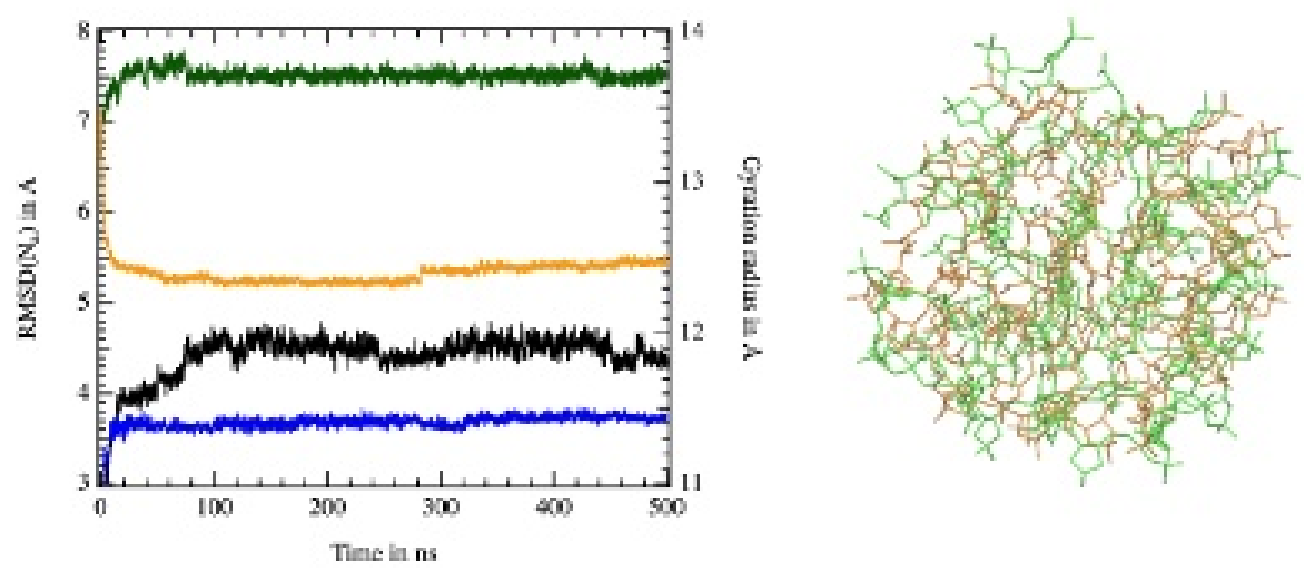

Figure 5: Left : temporal evolution of the P70 RMSD $\left(\mathrm{N}_{\mathrm{a}}\right)$ (left axis) and gyration radius (right axis) along the $500 n s$ simulations. Black (SRP) and blue (LRP) lines : $\operatorname{RMSD}\left(\mathrm{N}_{\mathrm{a}}\right)$ data. Green $(\mathbf{S R P})$ and orange $(\mathbf{L R P})$ lines : gyration radius data. Right : superposition of the starting (green) and final (orange) P70 CQS structures from the 500 ns SRP simulation. 


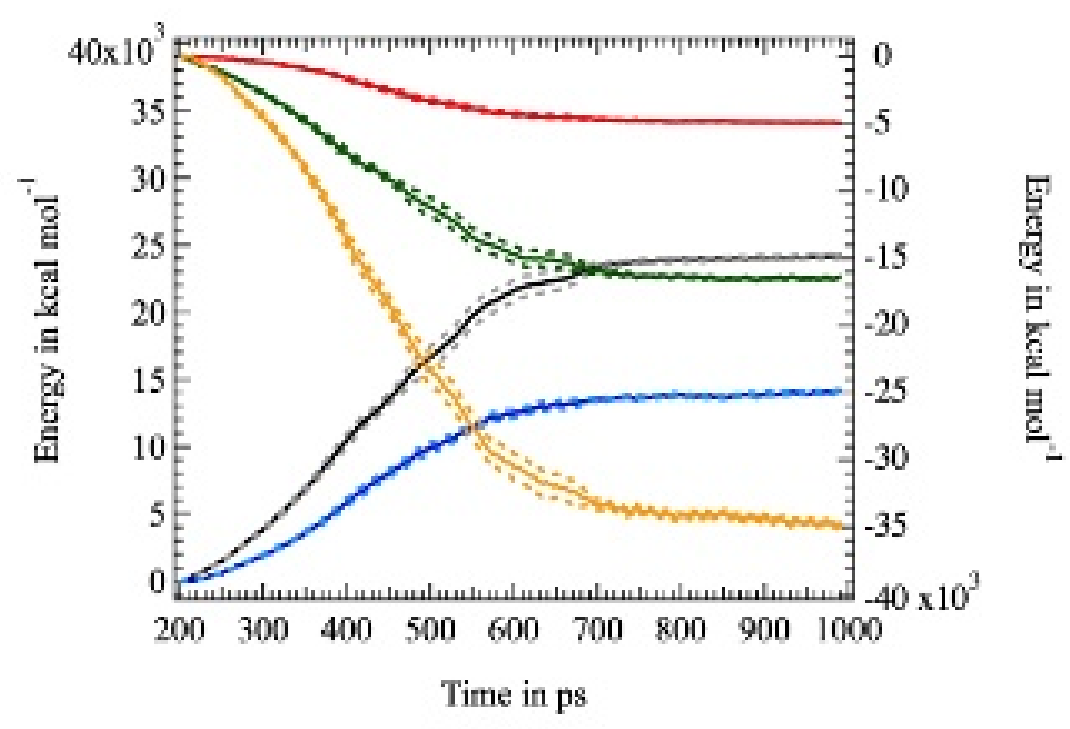

Figure 6: Temporal evolution of the energy component values computed by averaging data from all the independent simulations in the SRP medium, in bold lines. In dashed lines, the maximum deviations from the mean data. Black and blue : intraP70 and intra-counter ion cloud interaction energies (left axis) ; red, green and yellow : P70/PPPs and counter ions/PPPs polarization energies, and P70/counter ions interaction energy, respectively (right axis). All the energy components are shifted to be zero at simulation time $t=200 \mathrm{ps}$ at which the harmonic constraints preventing P70 structural transitions are removed. 


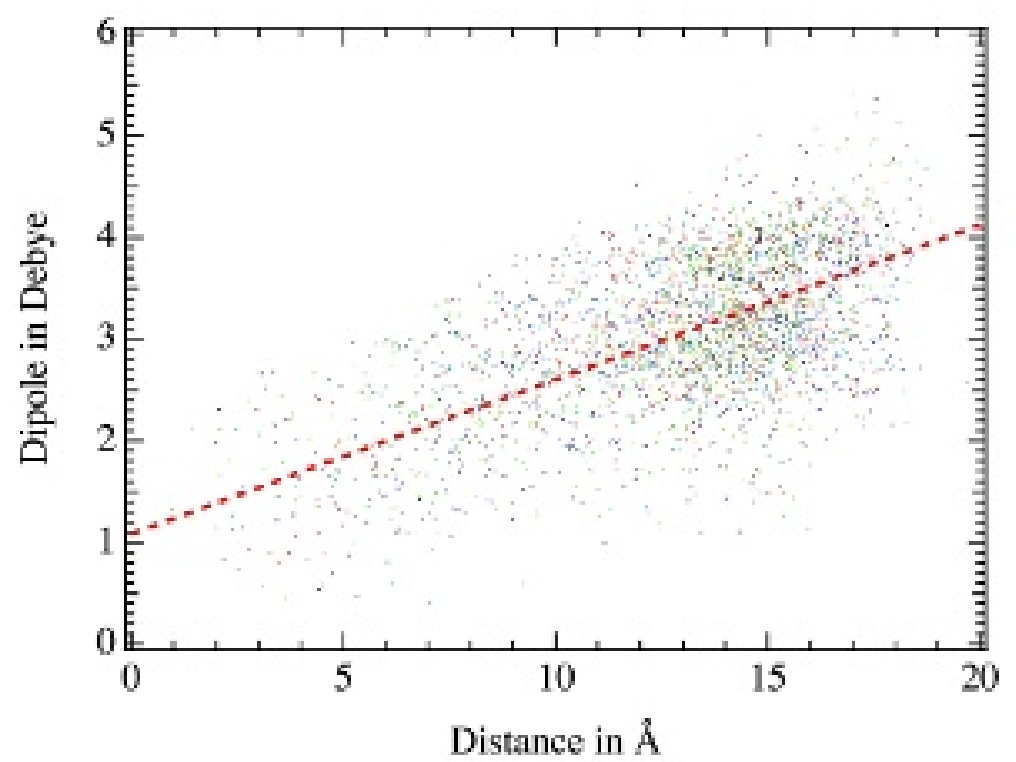

Figure 7: Mean induced dipole moment values of the $\mathbf{P} 70$ alkyl carbons computed along all the independent simulations as a function of the alkyl carbon distance to PCOM. The dashed line is the result of the linear regression fit (the regression coefficient is here of 0.6 ). 


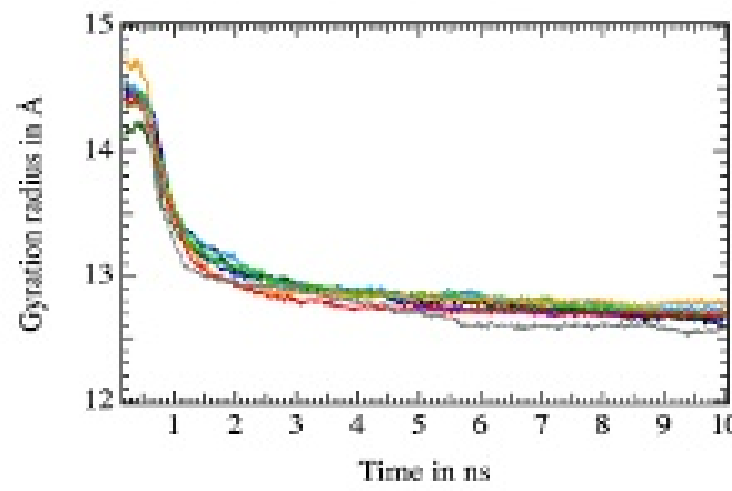

(a)

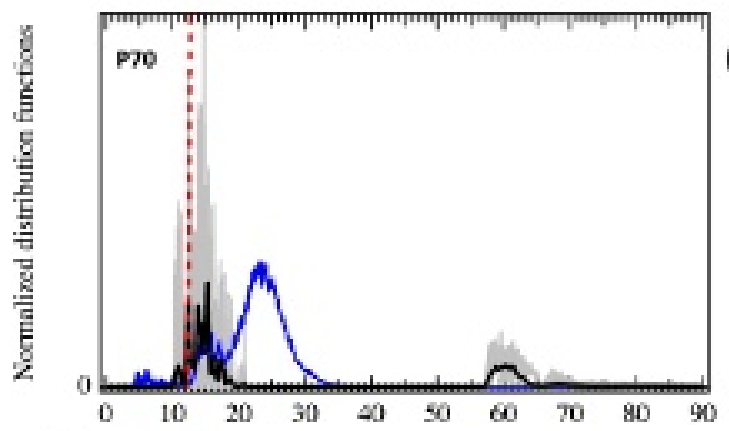

(b)

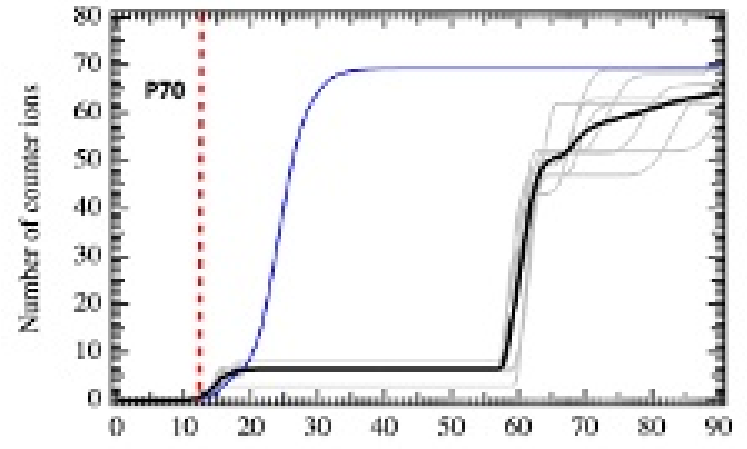

(c)

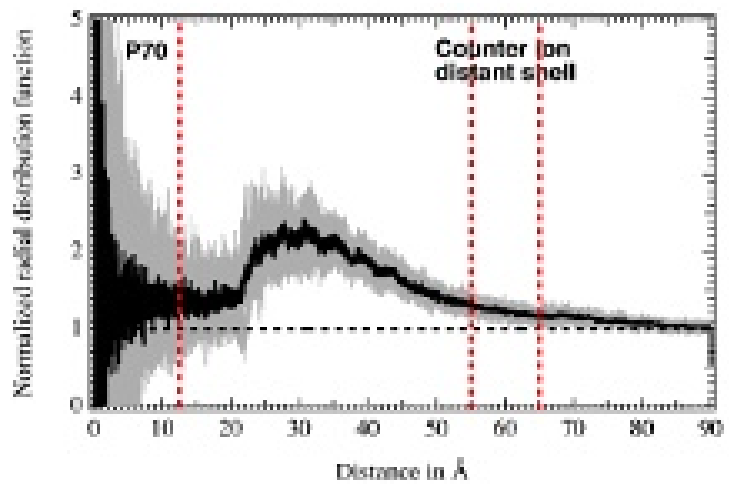

(d)

Figure 8: CQS P70 and counter ion structural data in a LRP medium. (a) Temporal evolution of the $\mathbf{P} 70$ gyration radius $R_{g}$ along the independent simulations. (b) and (c) : counter ion functions $g_{\mathrm{ci}}(r)$ and $N_{\mathrm{ci}}(r)$, respectively (black line : mean functions averaged over all the simulations; grey lines : data from each simulation ; blue thin lines : mean functions from P70 SRP simulations; the vertical red line is located at the mean $\mathbf{P 7 0}$ gyration radius $R_{g}$ ). (d) : P70 PCOM/second order PPPs radial distribution functions from the independent simulations (in light grey lines) and the corresponding mean function (bold black line). 

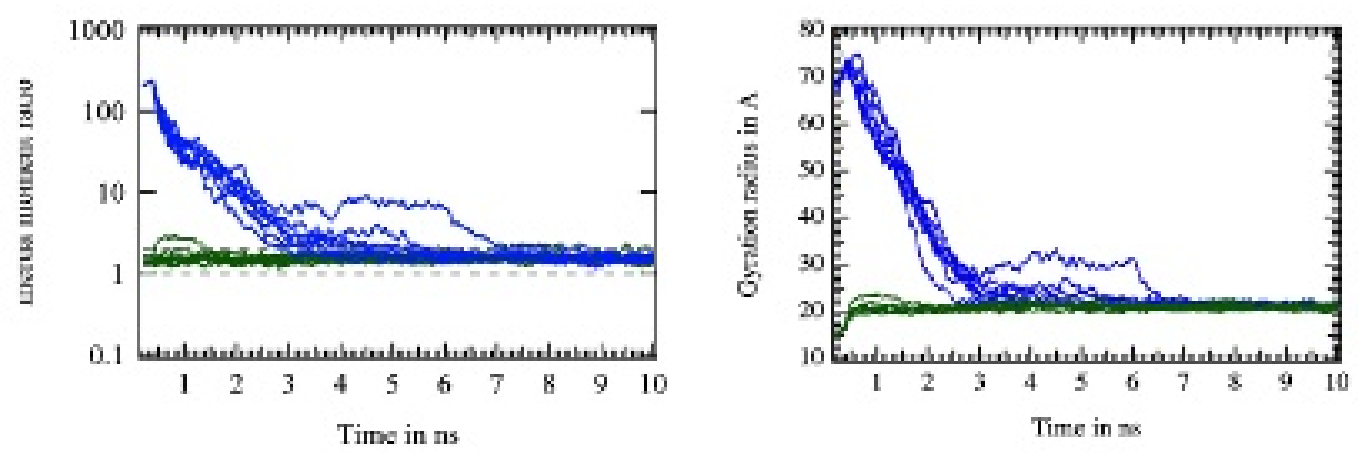

(a)
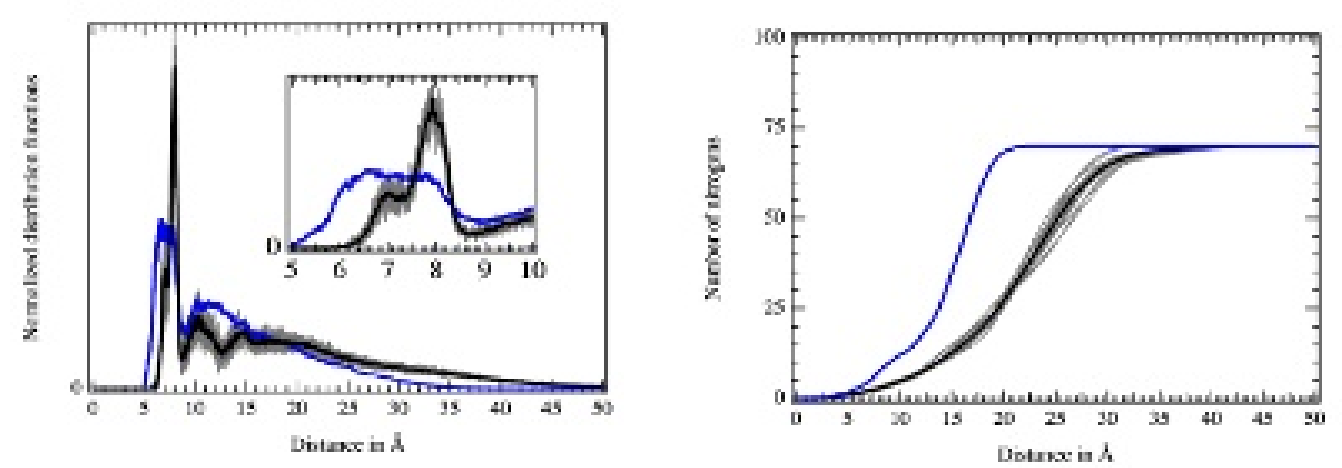

(b)
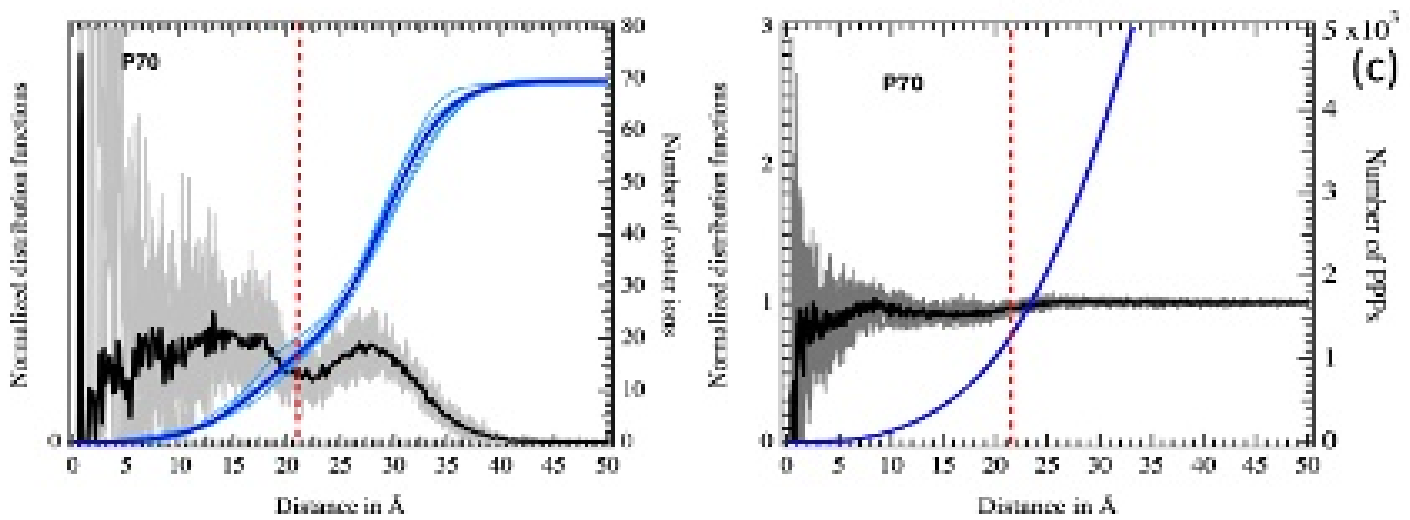

Figure 9: P70, counter ion and PPP structural data from non-polarizable P70 simulations in the SRP medium. (a) Temporal evolution of the $\mathbf{P 7 0}$ ratio $R_{\text {inertia }}$ (left) and gyration radius $R_{g}$ (right). Blue data : from the starting LSS structure; green data : from the starting CQS one. (b) Functions $g_{\mathrm{NN}}(r)$ (left) and $N_{\mathrm{N}_{\mathrm{a}}}(r)$ (right). Black line : data averaged over all the simulations; grey lines : data from each single simulation; blue line : mean functions from polarizable P70 simulations in SRP. (c) Left : Counter ion functions $g_{\mathrm{ci}}(r)$ (black) and $N_{\mathrm{ci}}(r)$ (blue), and right : PPP functions $g_{\mathrm{PPP}}(r)$ (black) and $N_{\mathrm{PPP}}(r)$ (blue). Bold lines : data averaged over all the simulations; thin lines : data from each simulation. The vertical red line is located at the mean $\mathbf{P} 70$ gyration radius $R_{g}$. 

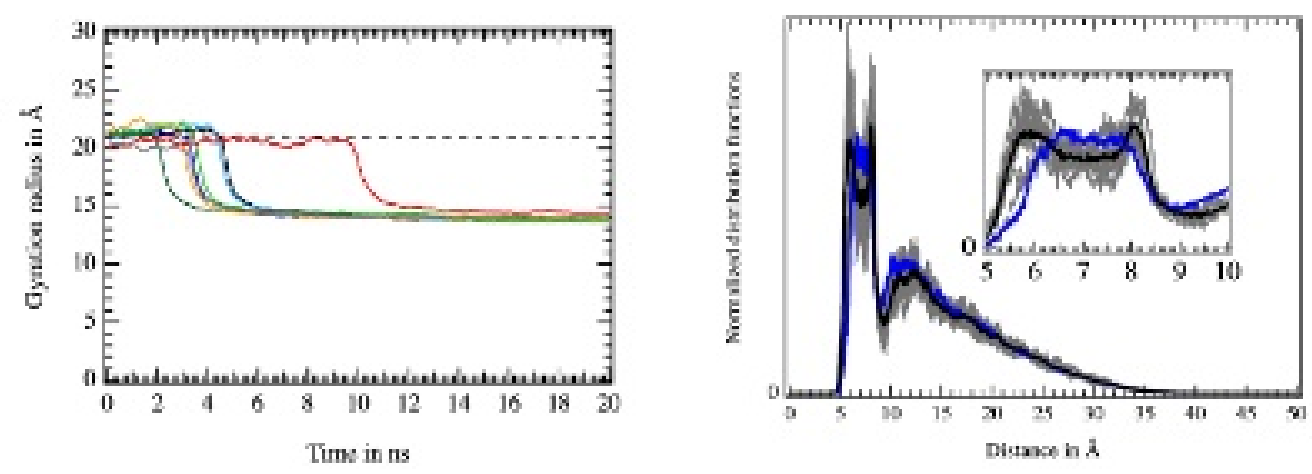

(a)
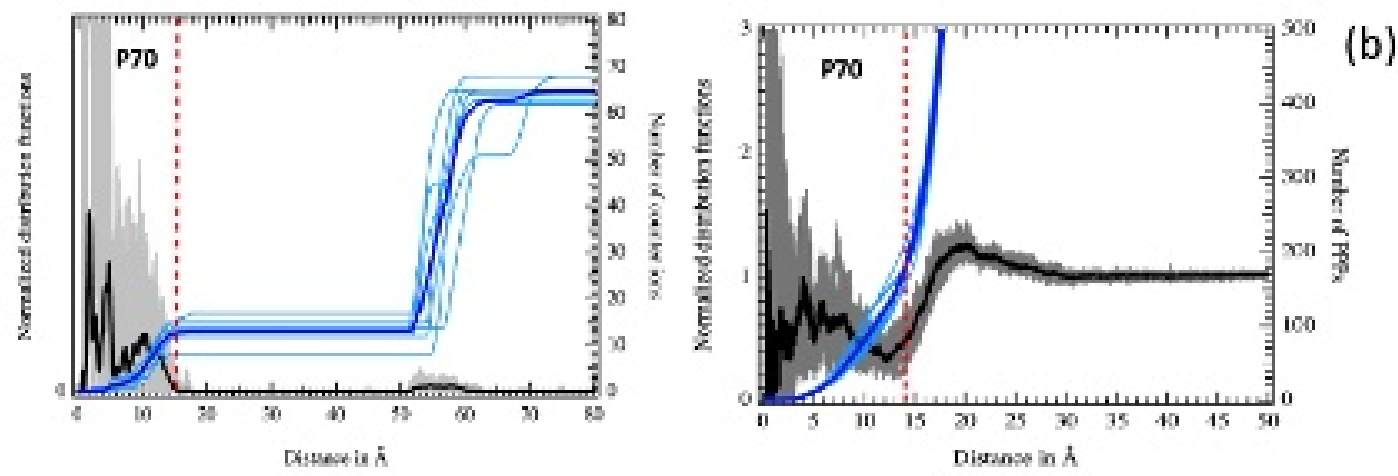

Figure 10: P70, counter ion and PPP structural data from non-polarizable P70 simulations in the LRP medium. (a) Left : temporal evolution of the P70 gyration radius $R_{g}$ (the horizontal dashed line is located at the non-polarizable $\mathbf{P} 70$ gyration radius converged value in $\mathbf{S R P}$ ). Right : functions $g_{\mathrm{NN}}(r)$ (black line : data averaged over all the LRP simulations; grey lines : data from each single LRP simulation; blue line : data averaged over the polarizable P70 simulations in SRP). (b) Left : counter ion functions $g_{\mathrm{ci}}(r)$ (black) and $N_{\mathrm{ci}}(r)$ (blue). Right : PPP functions $g_{\mathrm{PPP}}(r)$ (black) and $N_{\mathrm{PPP}}(r)$ (blue). Bold lines : data averaged over all the simulations; thin lines : data from each simulation. The vertical red line is located at the mean P70 gyration radius $R_{g}$. 


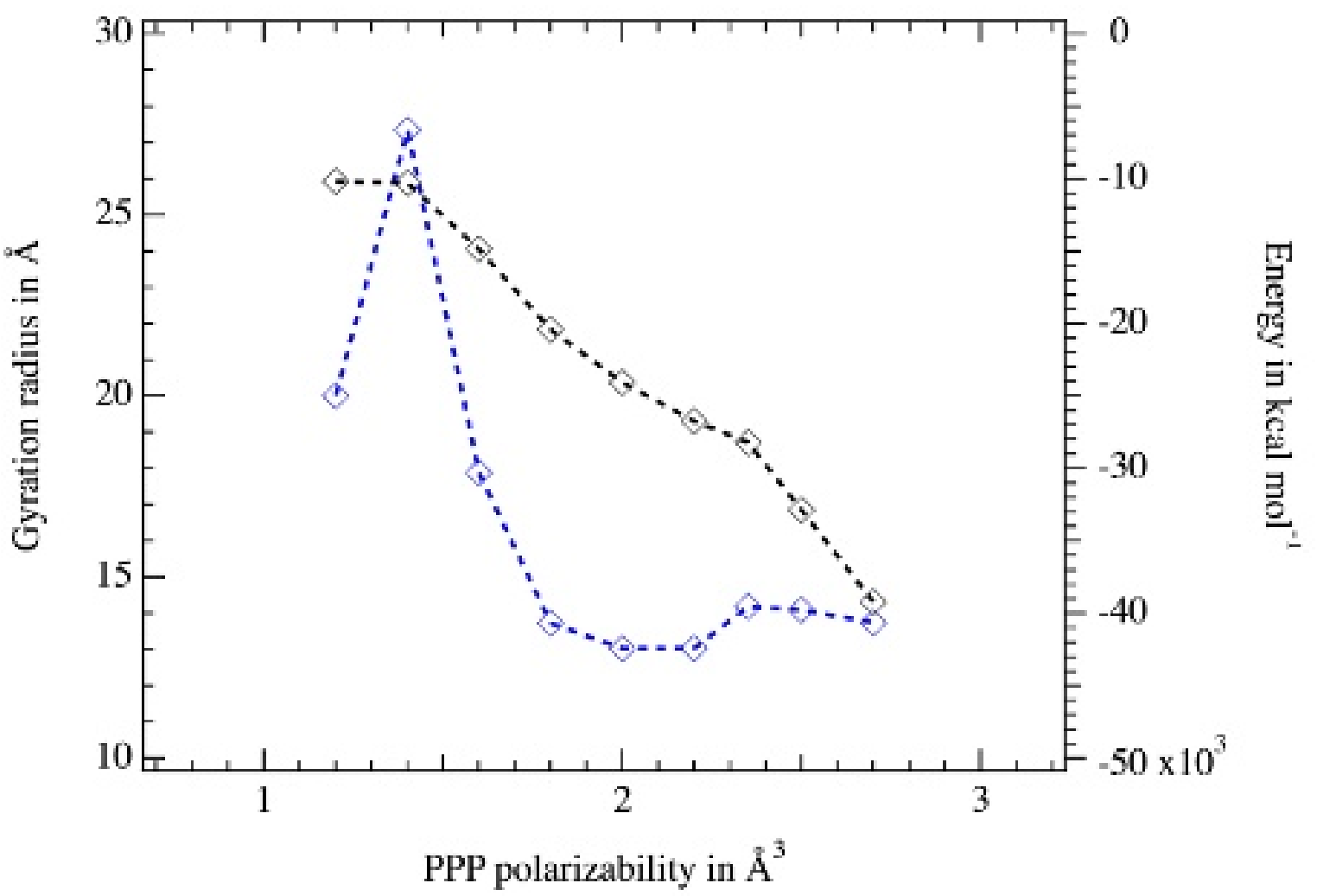

Figure 11: Mean P70 gyration radius $R_{g}$ (in blue and left axis) and mean P70/solvent interaction energy $U_{\mathrm{ps}}$ (in black and right axis) as a function of the PPP polarizability $\alpha_{s}$. That polarizability amounts to $2.35 \AA^{3}$ according to Equation (4). 


\section{References}

(1) Fernandez-Pena, L.; Guzman, E.; Léonforte, F.; Serrano-Pueyo, A.; Regulski, K.; Tournier-Couturier, L.; Ortega, F.; R.G., R.; Luengo, G. Effect of molecular structure of eco-friendly glycolipid biosurfactants on the adsorption of hair-care conditioning polymers. Colloids and Surfaces B: Biointerfaces 2020, 185, 110578.

(2) Manoj Lalwani, S.; Eneh, C. I.; Lutkenhaus, J. L. Emerging Trends in the Dynamics of Polyelectrolyte Complexes. Phys. Chem. Chem. Phys. 2020, 22, $24157-24177$.

(3) Gu, Y.; Zhao, J.; Johnson, J. A. Polymer Networks: From Plastics and Gels to Porous Frameworks. Angewandte Chemie International Edition 2020, 59, $5022-5049$.

(4) Minko, S.; Kiriy, A.; Gorodyska, G.; Stamm, M. Single Flexible Hydrophobic Polyelectrolyte Molecules Adsorbed on Solid Substrate: Transition between a Stretched Chain, Necklace-like Conformation and a Globule. Journal of the American Chemical Society 2002, 124, 3218-3219.

(5) Kiriy, A.; Gorodyska, G.; Minko, S.; Jaeger, W.; Štěpánek, P.; Stamm, M. Cascade of Coil-Globule Conformational Transitions of Single Flexible Polyelectrolyte Molecules in Poor Solvent. Journal of the American Chemical Society 2002, 124, 13454-13462.

(6) Kirwan, L. J.; Papastavrou, G.; Borkovec, M.; Behrens, S. H. Imaging the Coilto-Globule Conformational Transition of a Weak Polyelectrolyte by Tuning the Polyelectrolyte Charge Density. Nano Letters 2004, 4, 149-152.

(7) Xi, B.; Ran, S.-Y. Formation of DNA Pearl-Necklace Structures on Mica Sur- 
face Governed by Kinetics and Thermodynamics. Journal of Polymer Science Part B: Polymer Physics 2017, 55, 971-979.

(8) Aseyev, V. O.; Klenin, S. I.; Tenhu, H.; Grillo, I.; Geissler, E. Neutron Scattering Studies of the Structure of a Polyelectrolyte Globule in a Water-Acetone Mixture. Macromolecules 2001, 34, 3706-3709.

(9) Essafi, W.; Spiteri, M.-N.; Williams, C.; Boue, F. Hydrophobic Polyelectrolytes in Better Polar Solvent. Structure and Chain Conformation As Seen by SAXS and SANS. Macromolecules 2009, 42, 9568-9580.

(10) Essafi, W.; Abdelli, A.; Bouajila, G.; Boué, F. Behavior of Hydrophobic Polyelectrolyte Solution in Mixed Aqueous/Organic Solvents Revealed by Neutron Scattering and Viscosimetry. The Journal of Physical Chemistry B 2012, 116, $13525-13537$.

(11) Ben Mahmoud, S.; Essafi, W.; Brûlet, A.; Boué, F. How Necklace Pearls Evolve in Hydrophobic Polyelectrolyte Chains under Good Solvent Addition: A SANS Study of the Conformation. Macromolecules 2018, 51, 9259-9275.

(12) Duan, C.; Li, W.; Wang, R. Conformation of a single polyelectrolyte in poor solvents. The Journal of Chemical Physics 2020, 153, 064901.

(13) Panagiotopoulos, A. Molecular Simulation of Phase Equilibria: Simple, Ionic and Polymeric Fluids. Fluid Phase Equilibria 1992, 76, 97 - 112.

(14) Escobedo, F. A.; de Pablo, J. J. Molecular Simulation of Polymeric Networks and Gels: Phase Behavior and Swelling. Physics Reports 1999, 318, 85 - 112.

(15) Reddy, G.; Yethiraj, A. Implicit and Explicit Solvent Models for the Simulation of Dilute Polymer Solutions. Macromolecules 2006, 39, 8536-8542. 
(16) Chang, R.; Yethiraj, A. Dilute Solutions of Strongly Charged Flexible Polyelectrolytes in Poor Solvents: Molecular Dynamics Simulations with Explicit Solvent. Macromolecules 2006, 39, 821-828.

(17) Chremos, A.; Douglas, J. F. Polyelectrolyte Association and Solvation. The Journal of Chemical Physics 2018, 149, 163305.

(18) Chremos, A.; Horkay, F. Disappearance of the polyelectrolyte peak in salt-free solutions. Phys. Rev. E 2020, 102, 012611.

(19) Horkay, F.; Chremos, A.; Douglas, J. F.; L. Jones, R.; Lou, J.; Xia, Y. Systematic investigation of synthetic polyelectrolyte bottlebrush solutions by neutron and dynamic light scattering, osmometry, and molecular dynamics simulation. The Journal of Chemical Physics 2020, 152, 194904.

(20) Chremos, A.; Douglas, J. F. Polyelectrolyte Association and Solvation. The Journal of Chemical Physics 2018, 149, 163305.

(21) Mansel, B. W.; Irani, A. H.; Ryan, T. M.; McGillivray, D. J.; Chen, H.-L.; Williams, M. A. K. Resolving Solution Conformations of the Model SemiFlexible Polyelectrolyte Homogalacturonan Using Molecular Dynamics Simulations and Small-Angle X-ray scattering. The European Physical Journal E 2019, 42, 19 .

(22) Bacle, P.; Jardat, M.; Marry, V.; Mériguet, G.; Batôt, G.; Dahirel, V. CoarseGrained Models of Aqueous Solutions of Polyelectrolytes: Significance of Explicit Charges. The Journal of Physical Chemistry B 2020, 124, 288-301.

(23) Mintis, D. G.; Alexiou, T. S.; Mavrantzas, V. G. Effect of pH and Molecular Length on the Structure and Dynamics of Linear and Short-Chain Branched Poly(ethylene imine) in Dilute Solution: Scaling Laws from Detailed Molecular Dynamics Simulations. The Journal of Physical Chemistry B 2020, 124, 61546169 . 
(24) Jungwirth, P.; Tobias, D. J. Specific Ion Effects at the Air/Water Interface. Chemical Reviews 2006, 106, 1259-1281.

(25) Tobias, D. J.; Stern, A. C.; Baer, M. D.; Levin, Y.; Mundy, C. J. Simulation and Theory of Ions at Atmospherically Relevant Aqueous Liquid-Air Interfaces. Annual Review of Physical Chemistry 2013, 64, 339-359.

(26) Houriez, C.; Meot-Ner (Mautner), M.; Masella, M. Solvation of the Guanidinium Ion in Pure Aqueous Environments: A Theoretical Study from an Ab Initio-Based Polarizable Force Field. The Journal of Physical Chemistry B 2017, 121, 11219-11228.

(27) Li, P.; Merz, K. M. Metal Ion Modeling Using Classical Mechanics. Chemical Reviews 2017, 117, 1564-1686.

(28) McDaniel, J. G.; Yethiraj, A. Influence of Electronic Polarization on the Structure of Ionic Liquids. The Journal of Physical Chemistry Letters 2018, 9, 47654770.

(29) Bedrov, D.; Piquemal, J.-P.; Borodin, O.; MacKerell, A. D.; Roux, B.; Schräder, C. Molecular Dynamics Simulations of Ionic Liquids and Electrolytes Using Polarizable Force Fields. Chemical Reviews 2019, 119, 7940-7995.

(30) Huang, J.; Lopes, P. E. M.; Roux, B.; MacKerell, A. D. Recent Advances in Polarizable Force Fields for Macromolecules: Microsecond Simulations of Proteins Using the Classical Drude Oscillator Model. The Journal of Physical Chemistry Letters 2014, 5, 3144-3150.

(31) Masella, M.; Cuniasse, P. A Many-Body Model to Study Proteins. I. Applications to MLnm + Complexes, $\mathrm{Mm}+=\mathrm{Li}+, \mathrm{Na}+, \mathrm{K}+, \mathrm{Mg} 2+, \mathrm{Ca} 2+$, and $\mathrm{Zn} 2+$, $\mathrm{L}=\mathrm{H} 2 \mathrm{O}, \mathrm{CH} 3 \mathrm{OH}, \mathrm{HCONH} 2, \mathrm{n}=1-6$, and to Small Hydrogen Bonded Systems. The Journal of Chemical Physics 2003, 119, 1866-1873. 
(32) Rupakheti, C.; Lamoureux, G.; MacKerell, A. D.; Roux, B. Statistical Mechanics of Polarizable Force Fields Based on Classical Drude Oscillators with Dynamical Propagation by the Dual-Thermostat Extended Lagrangian. The Journal of Chemical Physics 2020, 153, 114108.

(33) Vázquez-Montelongo, E. A.; Vázquez-Cervantes, J. e. E.; Cisneros, G. A. Current Status of AMOEBA-IL: A Multipolar/Polarizable Force Field for Ionic Liquids. International Journal of Molecular Sciences 2020, 21.

(34) Ha-Duong, T.; Phan, S.; Marchi, M.; Borgis, D. Electrostatic on Particles : Phenomenological and Orientational Density Functional Theory Approach. The Journal of Chemical Physics 2002, 117, 541-556.

(35) Masella, M.; Borgis, D.; Cuniasse, P. Combining a Polarizable Force-Field and a Coarse-Grained Polarizable Solvent Model. II. Accounting for Hydrophobic Effects. Journal of Computational Chemistry 2011, 32, 2664-2678.

(36) Masella, M.; Borgis, D.; Cuniasse, P. A Multiscale Coarse-Grained Polarizable Solvent Model for Handling Long Tail Bulk Electrostatics. Journal of Computational Chemistry 2013, 34, 1112-1124.

(37) Masella, M.; Borgis, D.; Cuniasse, P. Combining a Polarizable Force-Field and a Coarse-Grained Polarizable Solvent Model: Application to Long Dynamics Simulations of Bovine Pancreatic Trypsin Inhibitor. Journal of Computational Chemistry 2008, 29, 1707-1724.

(38) Somasundaran, P.; Chakraborty, S.; Qiang, Q.; Deo, P.; Wang, J.; Zhang, R. Surfactants, Polymers and their Nanoparticles for Personal Care Applications. J Cosmet Sci. 2004, 55, S1-17.

(39) Cumming, J.; Hawker, D.; Chapman, H.; Nugent, K. Water, Air and Soil Pollution 2011, 216, 441-450. 
(40) Pahal, S.; Gakhar, R.; Raichur, A. M.; Varma, M. M. Polyelectrolyte multilayers for bio-applications: recent advancements. IET Nanobiotechnology 2017, 11, 903-908.

(41) Besha, A. T.; Tsehaye, M. T.; Aili, D.; Zhang, W.; Tufa, R. A. Design of Monovalent Ion Selective Membranes for Reducing the Impacts of Multivalent Ions in Reverse Electrodialysis. Membranes 2019, 10, 7.

(42) Beaugeard, V.; Muller, J.; Graillot, A.; Ding, X.; Robin, J.-J.; Monge, S. Acidic Polymeric Sorbents for the Removal of Metallic Pollution in Water: A Review. Reactive and Functional Polymers 2020, 152, 104599.

(43) Lemkul, J. A.; MacKerell, A. D. Polarizable Force Field for DNA Based on the Classical Drude Oscillator: I. Refinement Using Quantum Mechanical Base Stacking and Conformational Energetics. Journal of Chemical Theory and Computation 2017, 13, 2053-2071.

(44) Zhang, C.; Lu, C.; Jing, Z.; Wu, C.; Piquemal, J.-P.; Ponder, J. W.; Ren, P. AMOEBA Polarizable Atomic Multipole Force Field for Nucleic Acids. Journal of Chemical Theory and Computation 2018, 14, 2084-2108.

(45) Inakollu, V. S.; Geerke, D. P.; Rowley, C. N.; Yu, H. Polarisable Force Fields: What do They Add in Biomolecular Simulations? Current Opinion in Structural Biology 2020, 61, 182 - 190, Theory and Simulation Macromolecular Assemblies.

(46) Singh, A. N.; Yethiraj, A. Driving Force for the Complexation of Charged Polypeptides. The Journal of Physical Chemistry B 2020, 124, 1285-1292.

(47) Sadman, K.; Wang, Q.; Chen, Y.; Keshavarz, B.; Jiang, Z.; Shull, K. R. Influence of Hydrophobicity on Polyelectrolyte Complexation. Macromolecules 2017, 50, 9417-9426. 
(48) Lopez, C. G.; Colby, R. H.; Cabral, J. T. Electrostatic and Hydrophobic Interactions in NaCMC Aqueous Solutions: Effect of Degree of Substitution. Macromolecules 2018, 51, 3165-3175.

(49) De Gennes, P.G.,; Pincus, P.,; Velasco, R.M.,; Brochard, F., Remarks on polyelectrolyte conformation. J. Phys. France 1976, 37, 1461-1473.

(50) Khokhlov, A. R. On the collapse of weakly charged polyelectrolytes. Journal of Physics A: Mathematical and General 1980, 13, 979-987.

(51) Dobrynin, A. V.; Rubinstein, M.; Obukhov, S. P. Cascade of Transitions of Polyelectrolytes in Poor Solvents. Macromolecules 1996, 29, 2974-2979.

(52) Muthukumar, M. Double Screening in Polyelectrolyte Solutions: Limiting Laws and Crossover Formulas. The Journal of Chemical Physics 1996, 105, 51835199.

(53) Muthukumar, M. 50th Anniversary Perspective: A Perspective on Polyelectrolyte Solutions. Macromolecules 2017, 50, 9528-9560.

(54) Spiteri, M. N.; Williams, C. E.; Boué, F. Pearl-Necklace-Like Chain Conformation of Hydrophobic Polyelectrolyte: a SANS Study of Partially Sulfonated Polystyrene in Water. Macromolecules 2007, 40, 6679-6691.

(55) Thole, B. Molecular Polarizabilities Calculated with a Modified Dipole Interaction. Chemical Physics 1981, 59, 341-350.

(56) Réal, F.; Vallet, V.; Flament, J.-P.; Masella, M. Revisiting a Many-Body Model for Water Based on a Single Polarizable Site. From Gas Phase Clusters to Liquid and Air/Liquid Water Systems. The Journal of Chemical Physics 2013, 139, 114502.

(57) Reed, A. E.; Weinstock, R. B.; Weinhold, F. Natural Population Analysis. The Journal of Chemical Physics 1985, 83, 735-746. 
(58) Houriez, C.; Meot-Ner (Mautner), M.; Masella, M. Simulated Solvation of Organic Ions: Protonated Methylamines in Water Nanodroplets. Convergence toward Bulk Properties and the Absolute Proton Solvation Enthalpy. The Journal of Physical Chemistry B 2014, 118, 6222-6233.

(59) Houriez, C.; Meot-Ner (Mautner), M.; Masella, M. Simulated Solvation of Organic Ions II: Study of Linear Alkylated Carboxylate Ions in Water Nanodrops and in Liquid Water. Propensity for Air/Water Interface and Convergence to Bulk Solvation Properties. The Journal of Physical Chemistry B 2015, 119, 12094-12107.

(60) Houriez, C.; Vallet, V.; Réal, F.; Meot-Ner (Mautner), M.; Masella, M. Organic Ion Association in Aqueous Phase and Ab Initio-Based Force Fields: The Case of Carboxylate/Ammonium Salts. The Journal of Chemical Physics 2017, 147, 161720.

(61) Li, A. H.-T.; Chao, S. D. Interaction Energies of Dispersion-Bound Methane Dimer from Coupled Cluster Method at Complete Basis Set Limit. Journal of Molecular Structure: THEOCHEM 2009, 897, 90 - 94.

(62) Chandler, D. Interfaces and the Driving Force of Hydrophobic Assembly. Nature 2005, 437, 640-647.

(63) Chremos, A.; Douglas, J. F. The Influence of Polymer and Ion Solvation on the Conformational Properties of Flexible Polyelectrolytes. Gels 2018, 4.

(64) http://biodev.cea.fr/polaris/download.html/.

(65) Cancès, E.; Legoll, F.; Stoltz, G. Theoretical and Numerical Comparison of Some Sampling Methods for Molecular Dynamics. ESAIM: M2AN 2007, 41, $351-389$. 
(66) Martyna, G. J.; Tuckerman, M. E.; Tobias, D. J.; Klein, M. L. Explicit Reversible Integrators for Extended Systems Dynamics. Mol. Phys. 1996, 87, $1117-1157$.

(67) Wu, H.; Ting, J. M.; Werba, O.; Meng, S.; Tirrell, M. V. Non-equilibrium phenomena and kinetic pathways in self-assembled polyelectrolyte complexes. The Journal of Chemical Physics 2018, 149, 163330.

(68) Guárdia, E.; Skarmoutsos, I.; Masia, M. On Ion and Molecular Polarization of Halides in Water. Journal of Chemical Theory and Computation 2009, 5, $1449-1453$.

(69) Réal, F.; Trumm, M.; Schimmelpfennig, B.; Masella, M.; Vallet, V. Further Insights in the Ability of Classical Nonadditive Potentials to Model Actinide Ion-Water Interactions. Journal of Computational Chemistry 2012, 34, 707719.

(70) Trumm, M.; Guerrero Martinez, Y. O.; Réal, F.; Schimmelpfennig, B.; Masella, M.; Vallet, V. Modeling the Hydration of Mono-Atomic Anions From the Gas Phase to the Bulk Phase: The Case of the Halide Ions $\mathrm{F}^{-}, \mathrm{Cl}^{-}$, and $\mathrm{Br}^{-}$. The Journal of Chemical Physics 2012, 136, 044509.

(71) Timko, J.; Bucher, D.; Kuyucak, S. Dissociation of NaCl in Water from Ab Initio Molecular Dynamics Simulations. The Journal of Chemical Physics 2010, 132, 114510.

(72) Luo, Y.; Jiang, W.; Yu, H.; MacKerell, A. D.; Roux, B. Simulation Study of Ion Pairing in Concentrated Aqueous Salt Solutions with a Polarizable Force Field. Faraday Discuss. 2013, 160, 135-149.

(73) Debiec, K. T.; Gronenborn, A. M.; Chong, L. T. Evaluating the Strength of Salt Bridges: A Comparison of Current Biomolecular Force Fields. The Journal of Physical Chemistry B 2014, 118, 6561-6569. 
(74) Baker, N. A.; Sept, D.; Joseph, S.; Holst, M. J.; McCammon, J. A. Electrostatics of Nanosystems: Application to Microtubules and the Ribosome. Proceedings of the National Academy of Sciences 2001, 98, 10037-10041.

(75) Houriez, C.; Réal, F.; Vallet, V.; Mautner, M.; Masella, M. Ion Hydration Free Energies and Water Surface Potential in Water nano Drops: The Cluster Pair Approximation and the Proton Hydration Gibbs Free Energy in Solution. The Journal of Chemical Physics 2019, 151, 174504.

(76) Georgalis, Y.; Kierzek, A. M.; Saenger, W. Cluster Formation in Aqueous Electrolyte Solutions Observed by Dynamic Light Scattering. The Journal of Physical Chemistry B 2000, 104, 3405-3406.

(77) Samal, S.; Geckeler, K. E. Unexpected Solute Aggregation in Water on Dilution. Chem. Commun. 2001, 2224-2225.

(78) Bharmoria, P.; Gupta, H.; Mohandas, V. P.; Ghosh, P. K.; Kumar, A. Temperature Invariance of $\mathrm{NaCl}$ Solubility in Water: Inferences from Salt-Water Cluster Behavior of $\mathrm{NaCl}, \mathrm{KCl}$, and $\mathrm{NH} 4 \mathrm{Cl}$. The Journal of Physical Chemistry B 2012, 116, 11712-11719.

(79) Konovalov, A. I.; Ryzhkina, I. S. Highly Diluted Aqueous Solutions: Formation of Nano-Sized Molecular Assemblies (Nanoassociates). Geochemistry International 2014, 52, 1207-1226.

(80) Pliego, J. R. The Role of Intermolecular Forces in Ionic Reactions: the Solvent Effect, Ion-Pairing, Aggregates and Structured Environment. Org. Biomol. Chem. 2021, 19, 1900-1914.

(81) Varghese, A.; Vemparala, S.; Rajesh, R. Phase Transitions of a Single Polyelectrolyte in a Poor Solvent with Explicit Counterions. The Journal of Chemical Physics 2011, 135, 154902. 
1177 (82) Coles, J. P.; Masella, M. The Fast Multipole Method and Point Dipole Moment $1178 \quad$ Polarizable Force Fields. The Journal of Chemical Physics 2015, 142, 024109. 


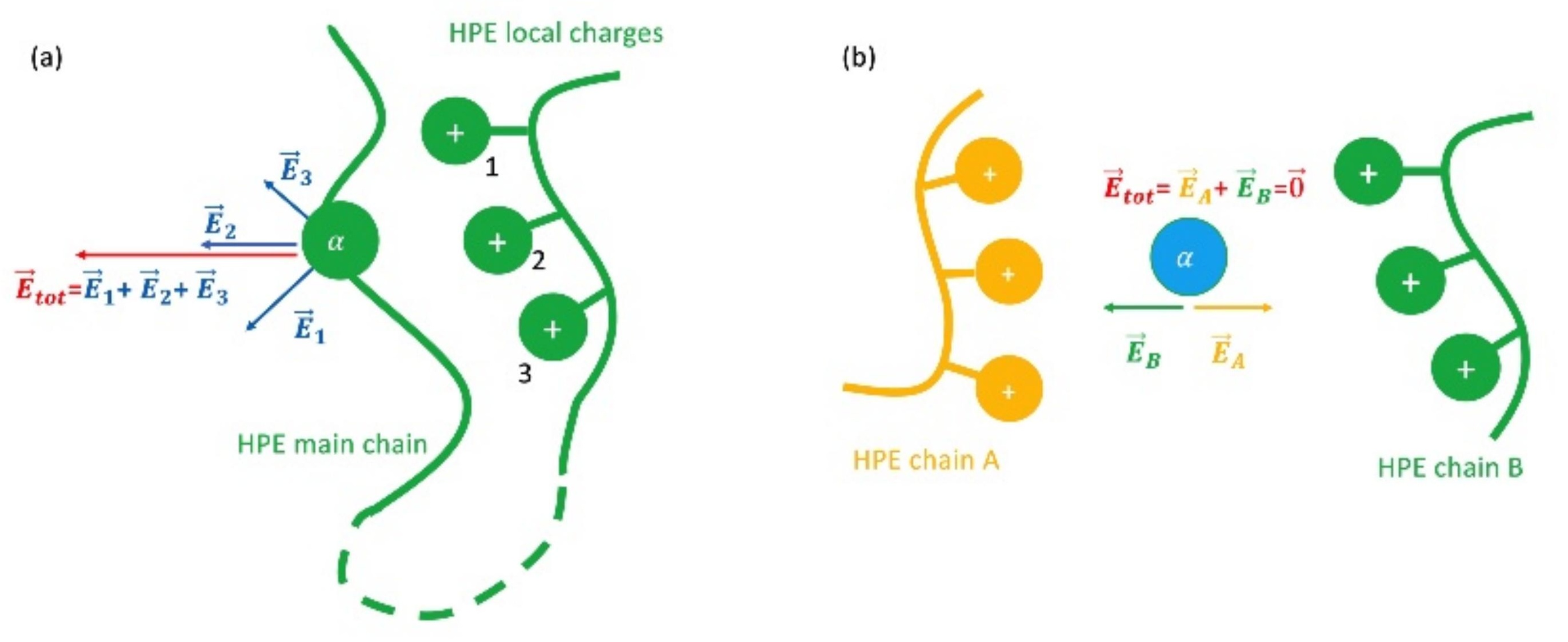




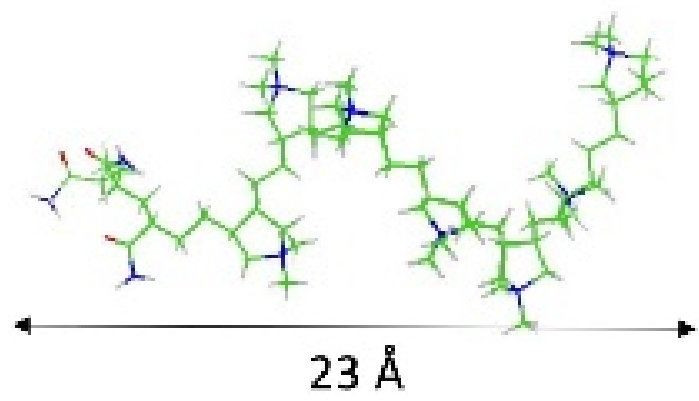

(a)

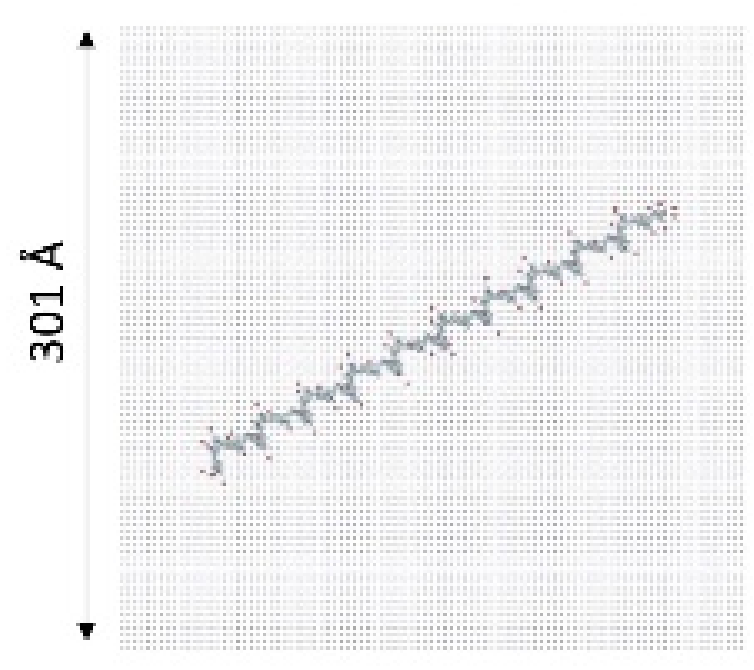

(b)

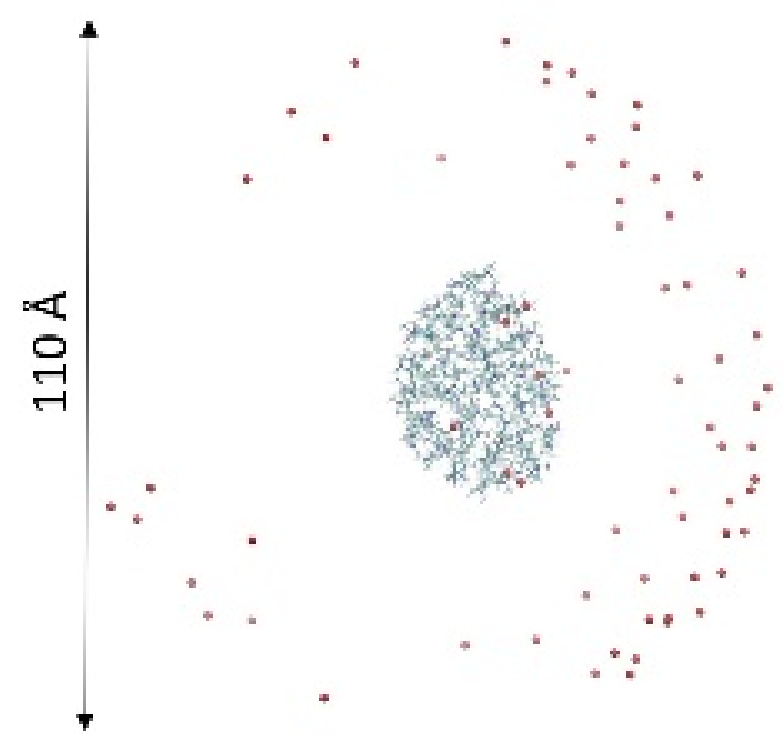

(c)

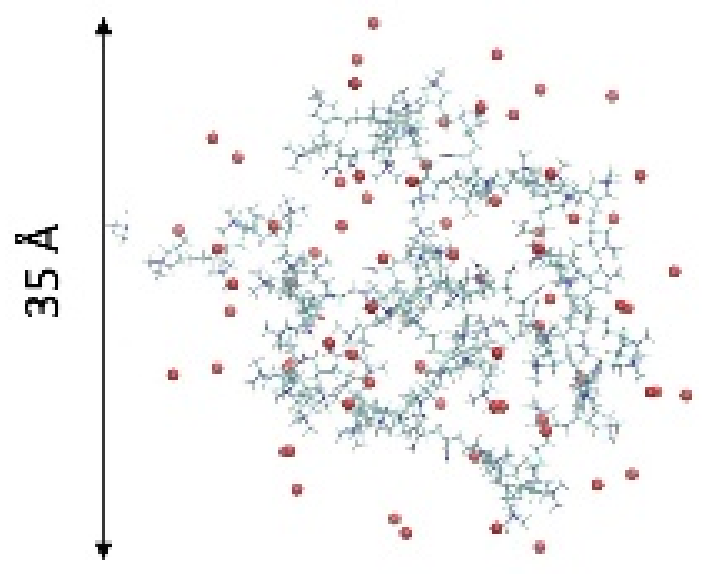


A
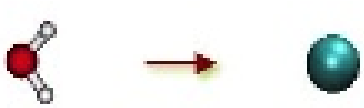

$\longrightarrow$
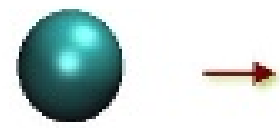

\section{Explicit water}

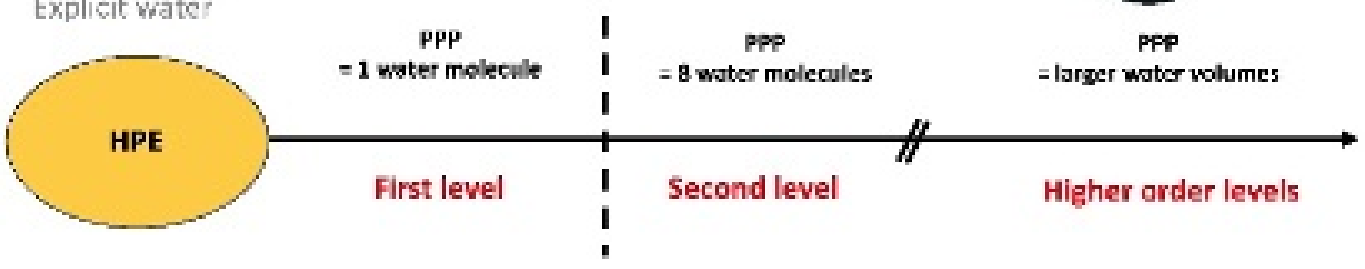

B

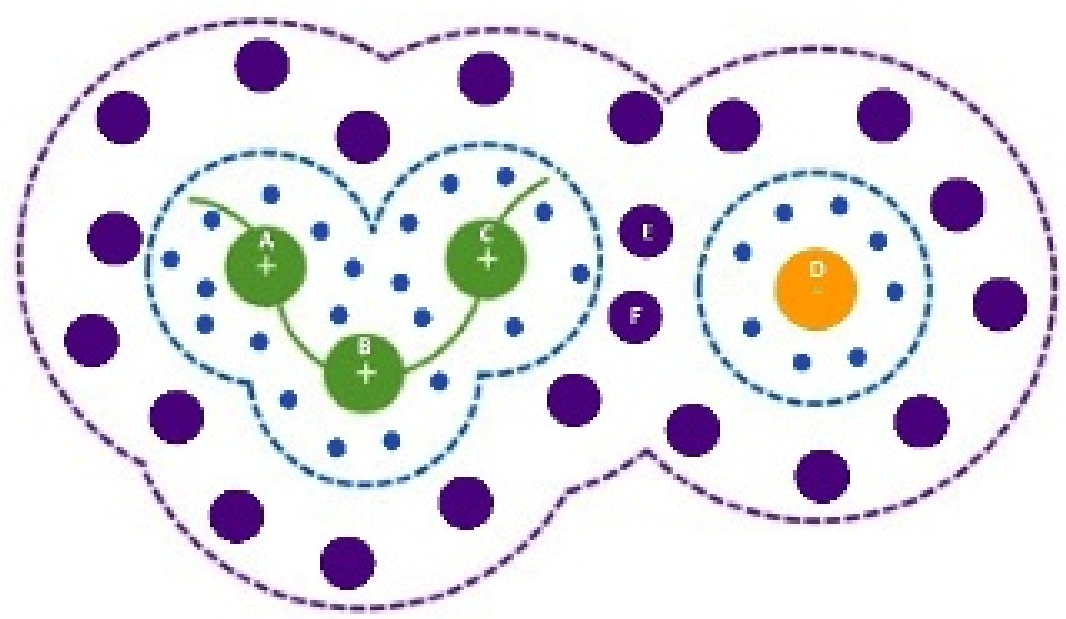



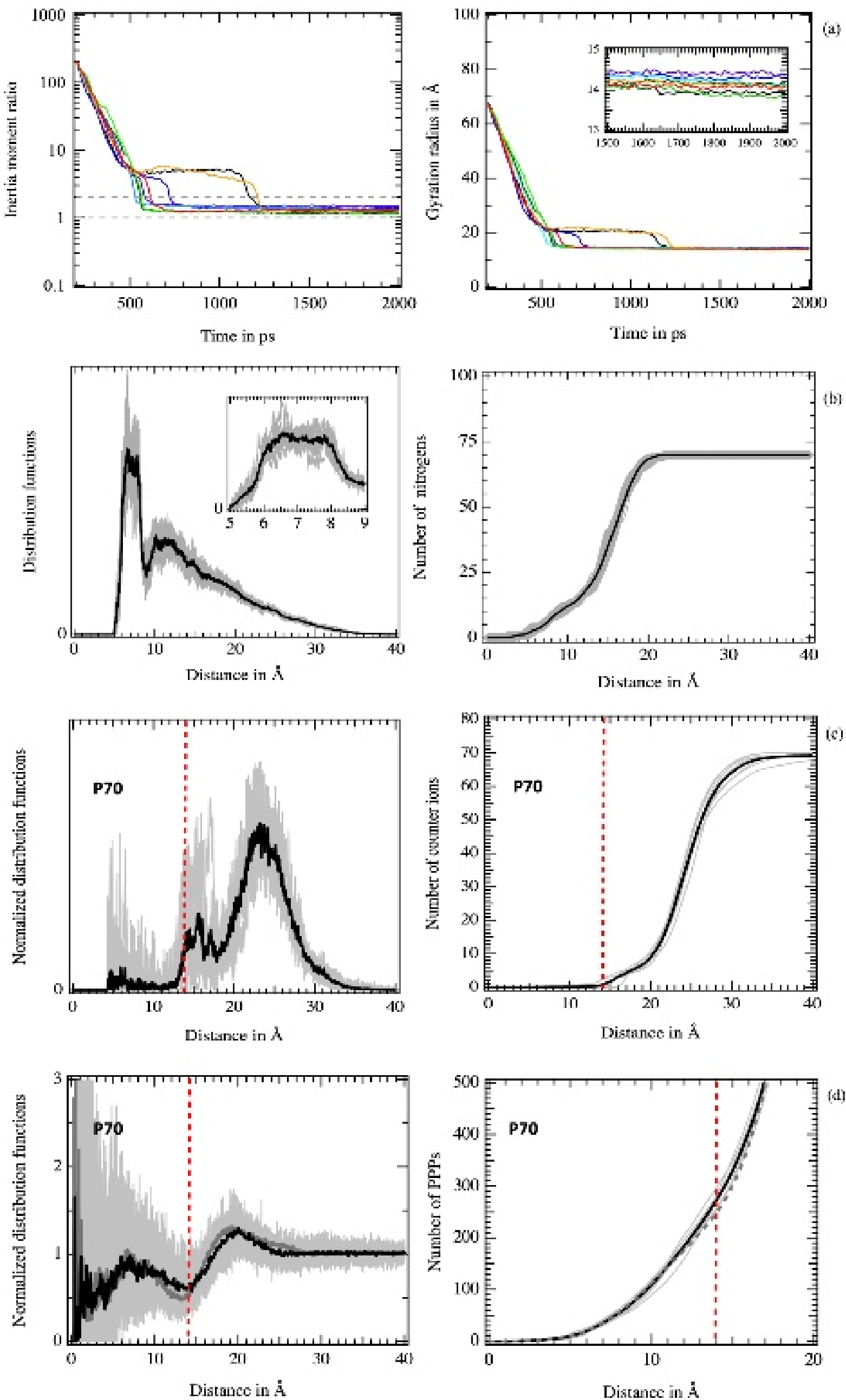


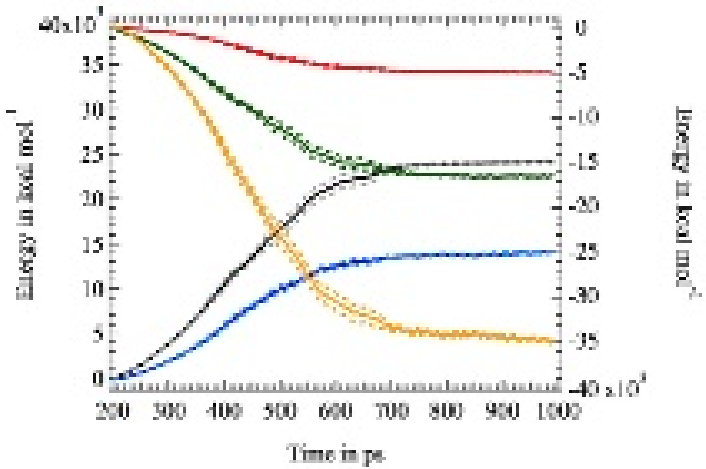




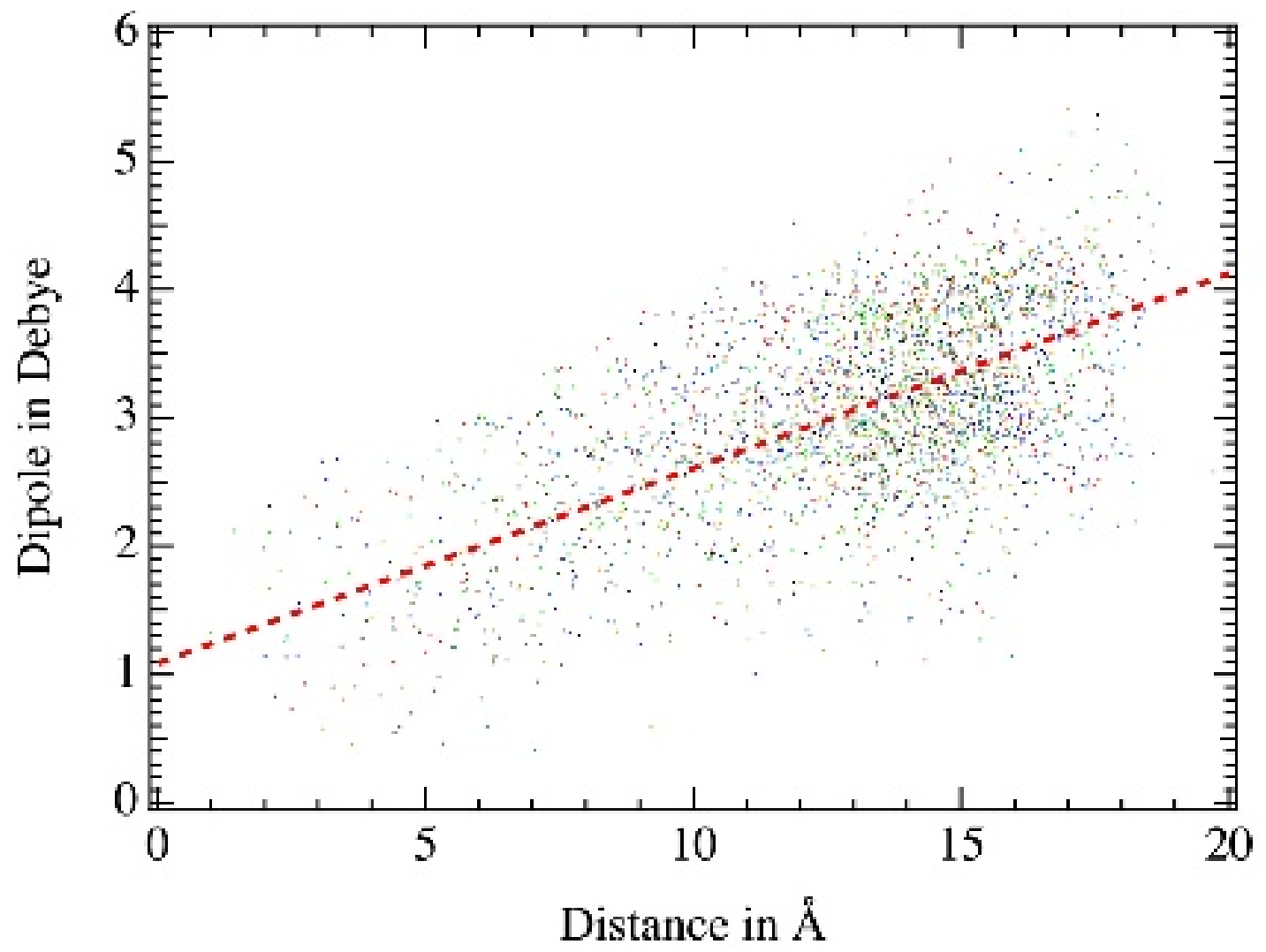




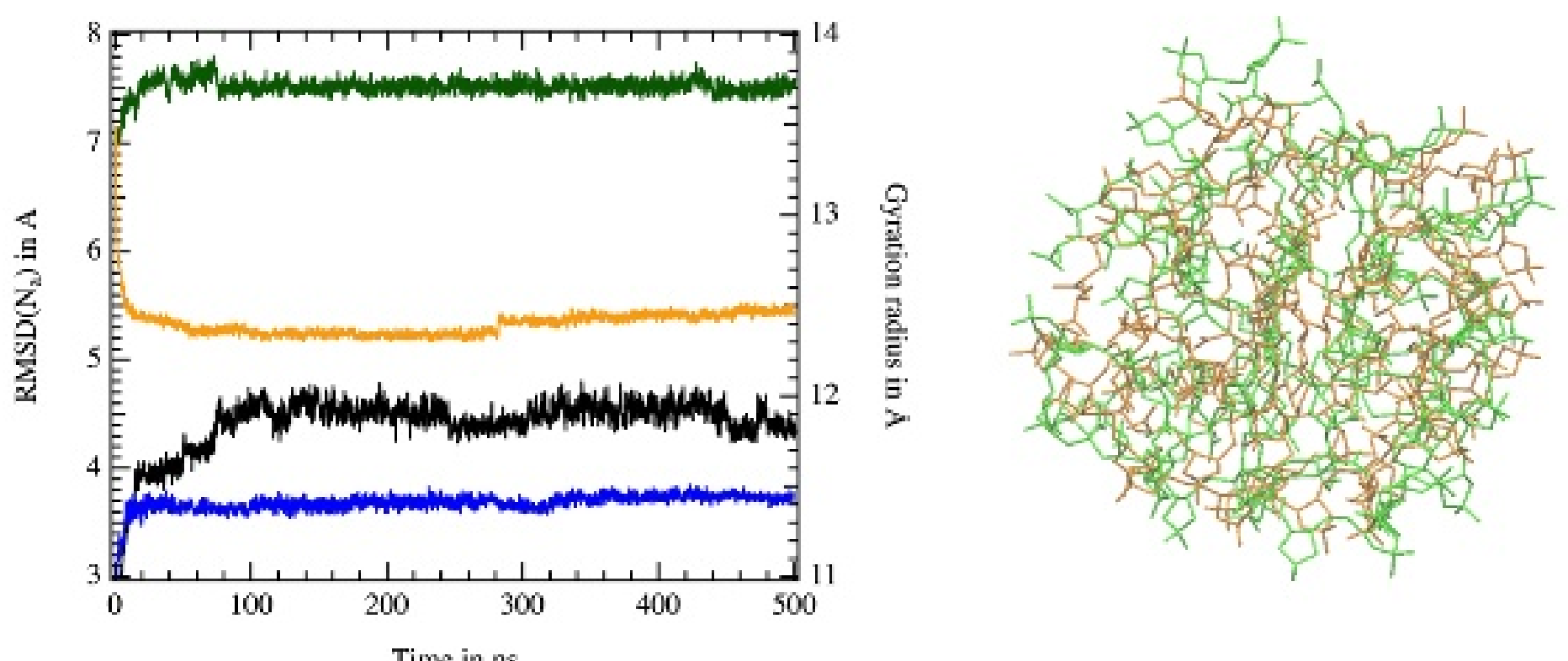

Time in ns 


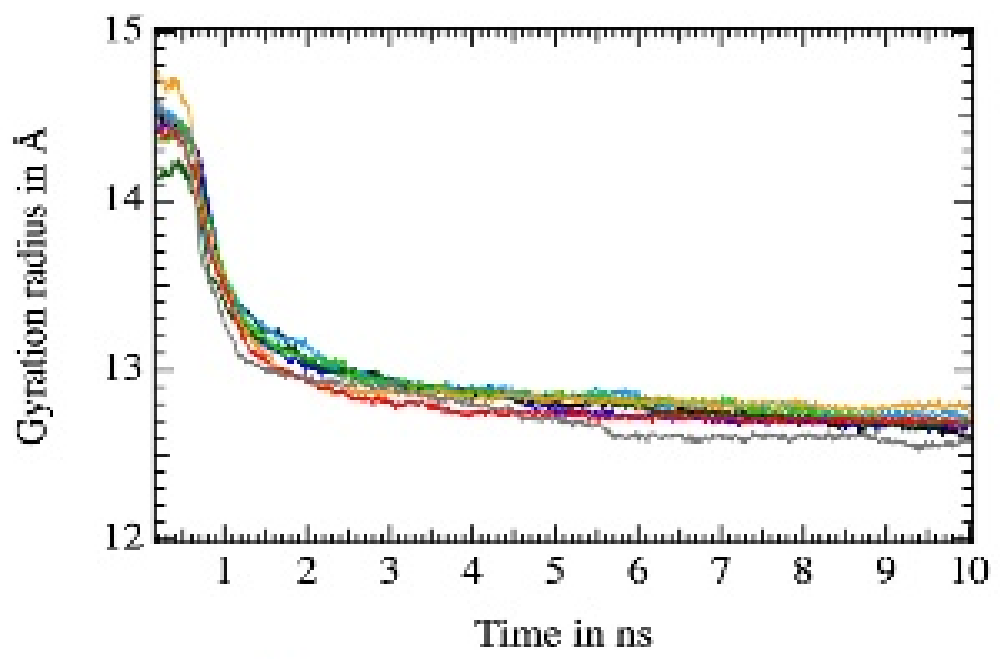

(a)

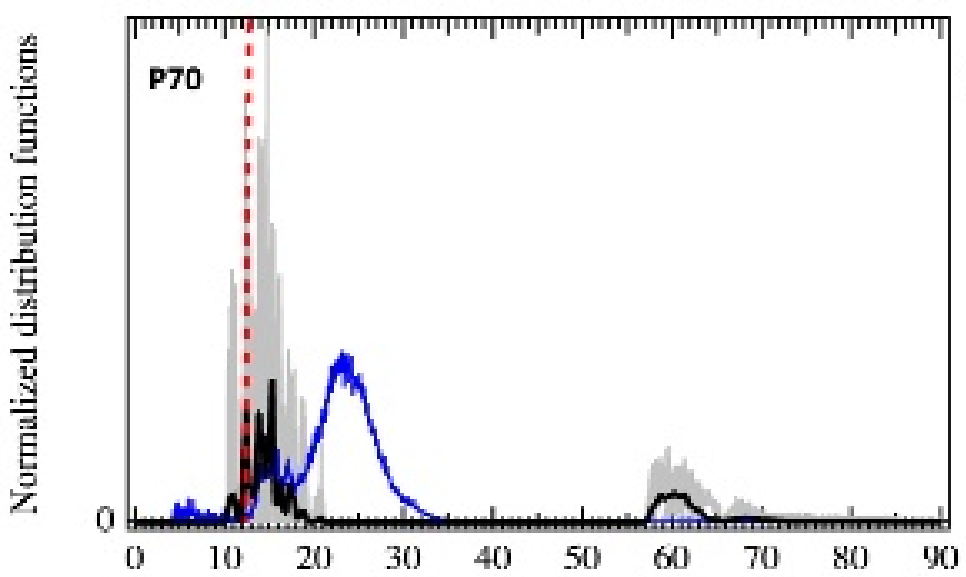

(b)

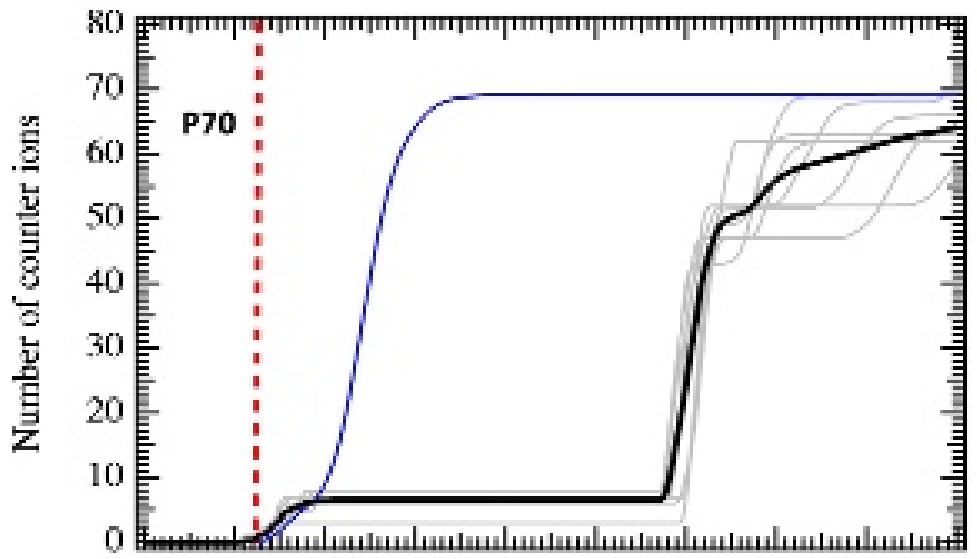

(c)

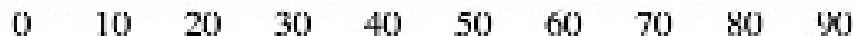

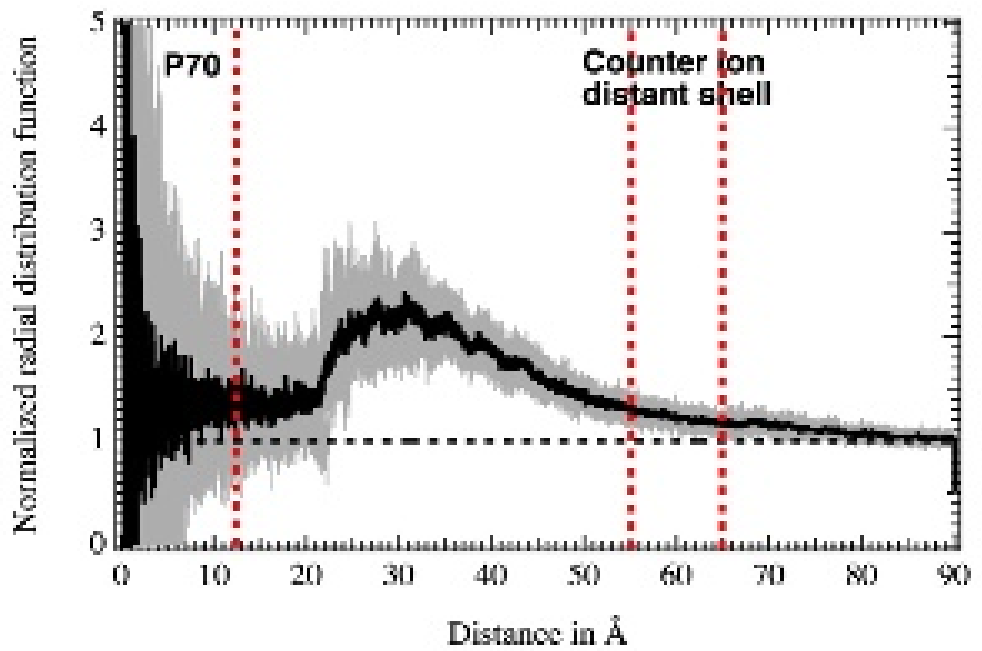




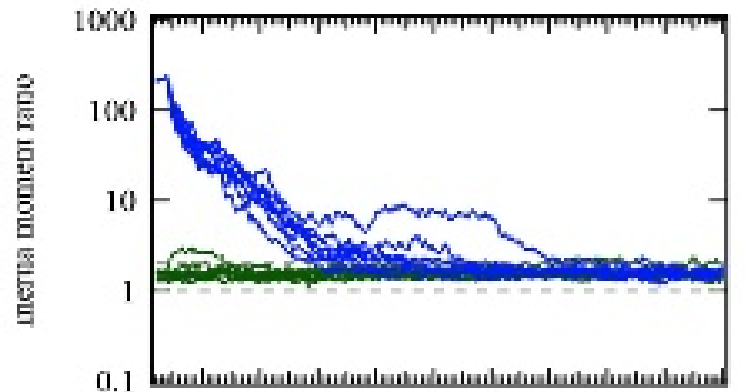

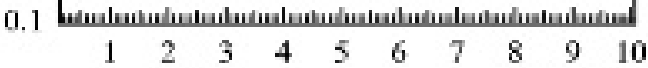

Time in as
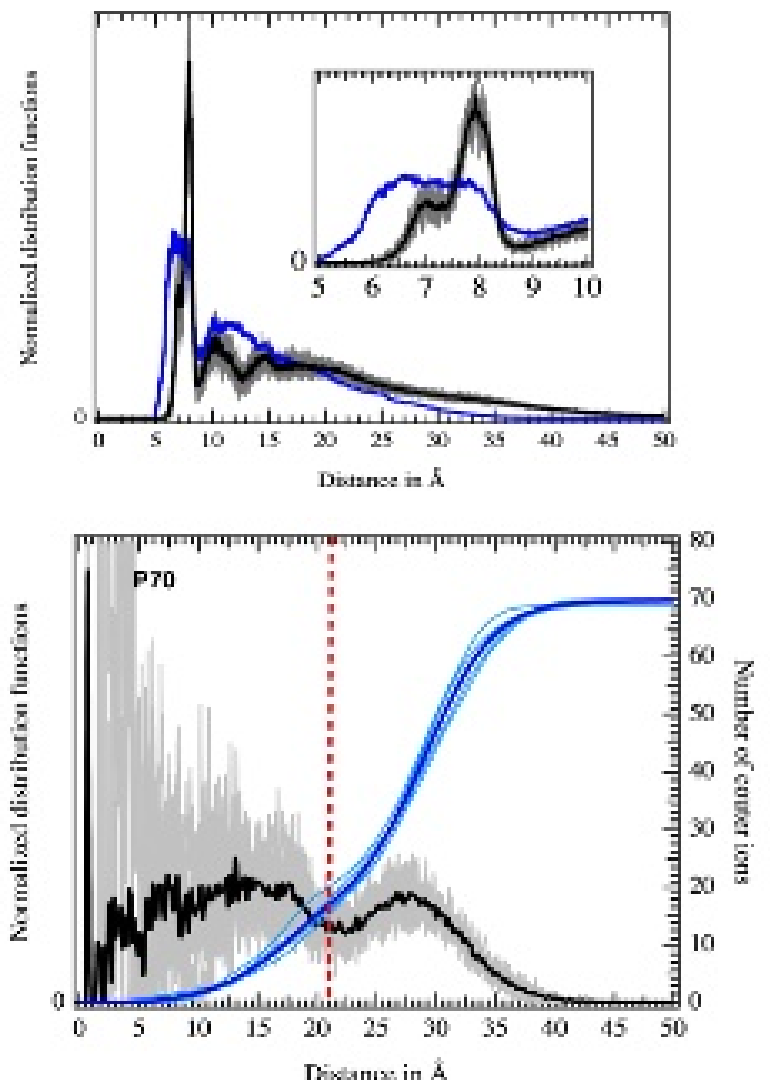

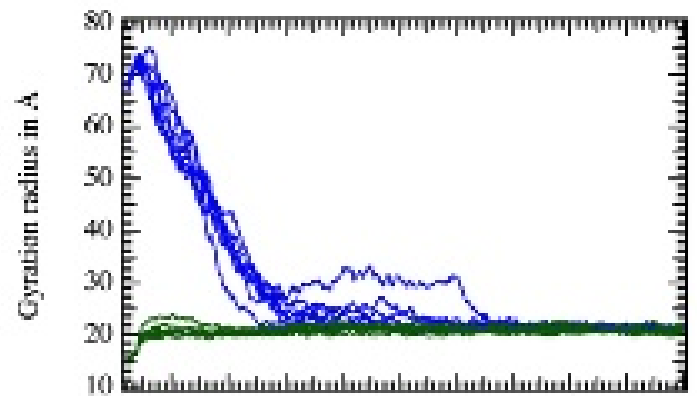

(a)

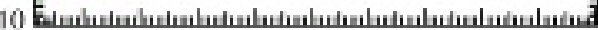

Time in ns

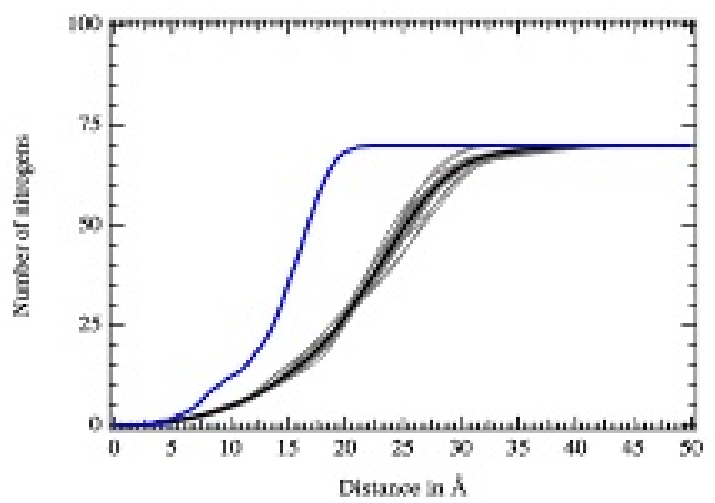

(b)

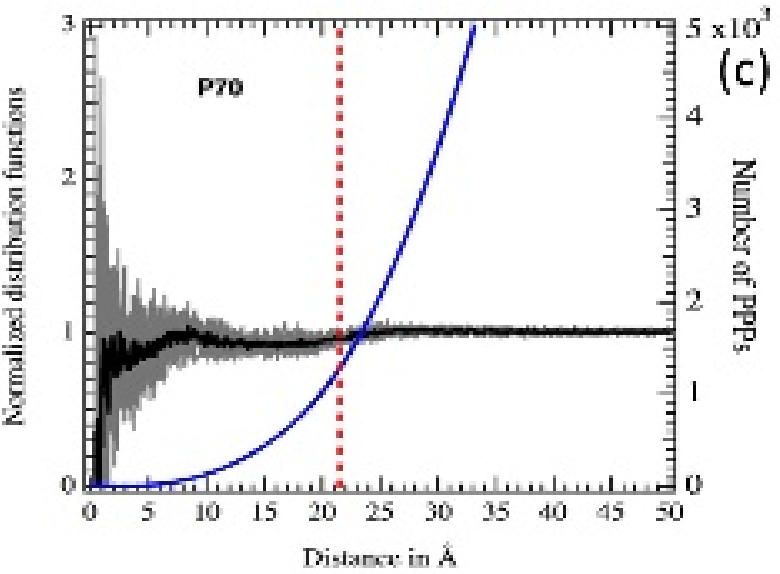



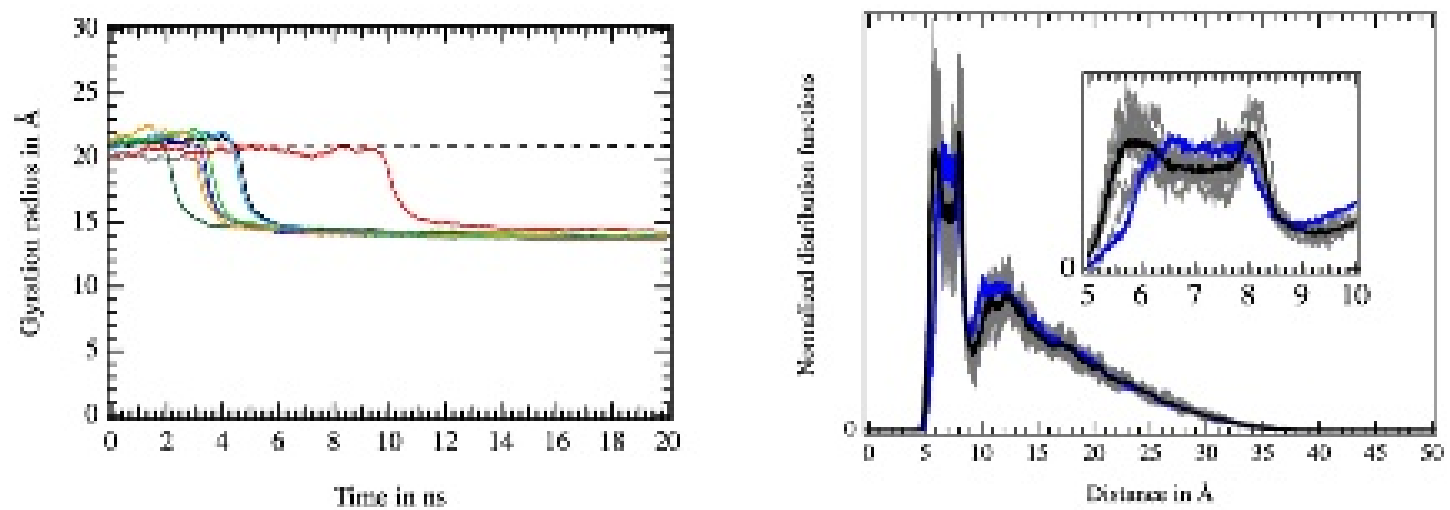

(a)
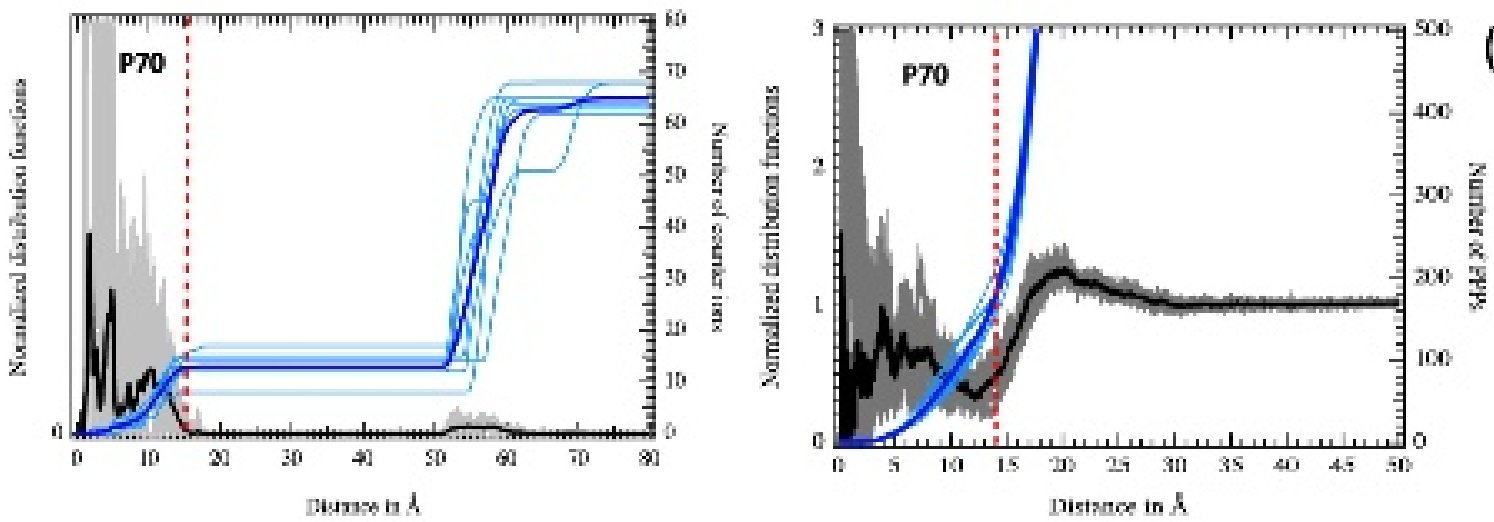

(b)

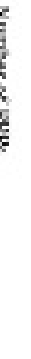




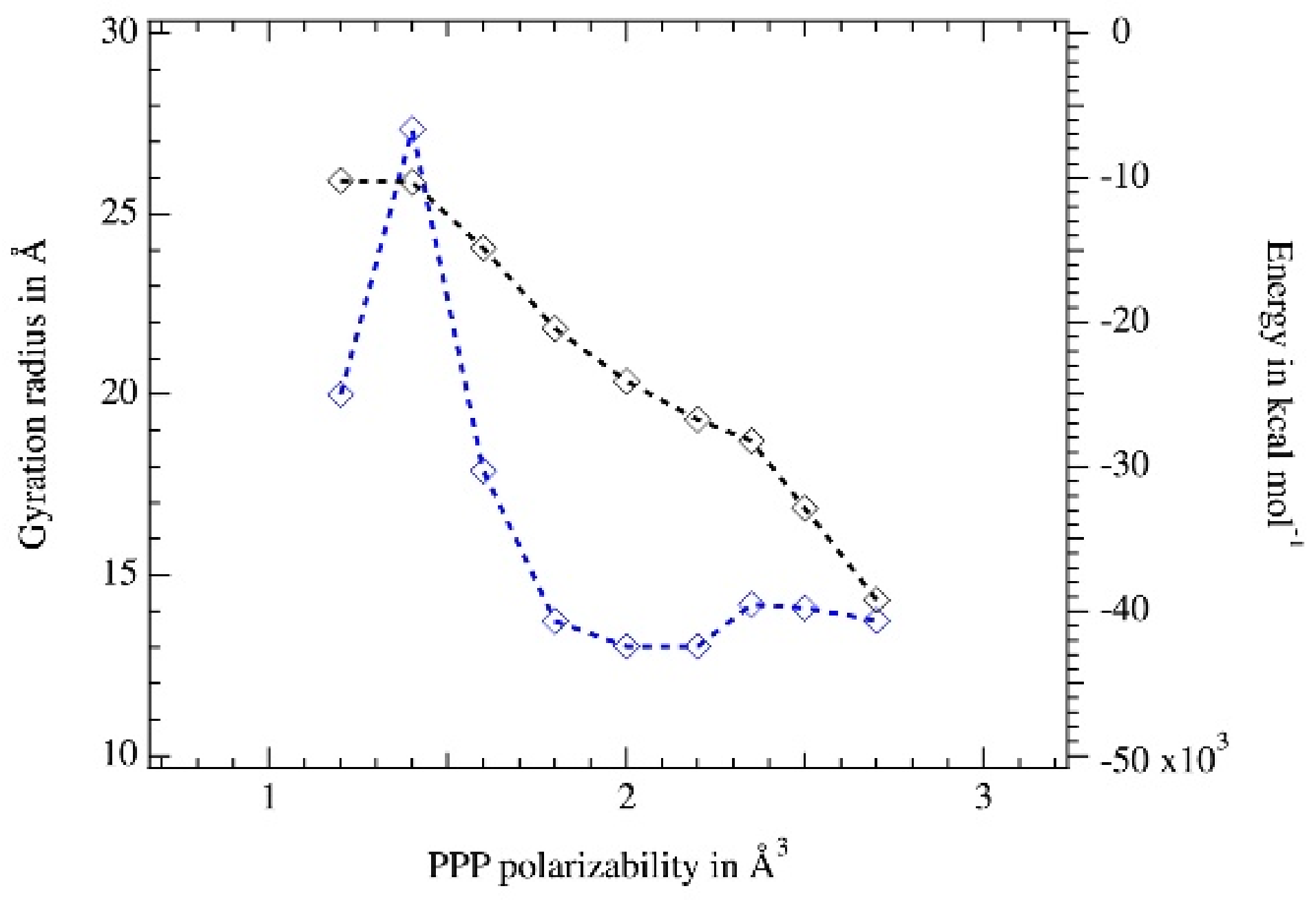

